\title{
HYDROCARBON LIQUID FLOW CALIBRATION SERVICE
}

NIST Special Publication 250-1039

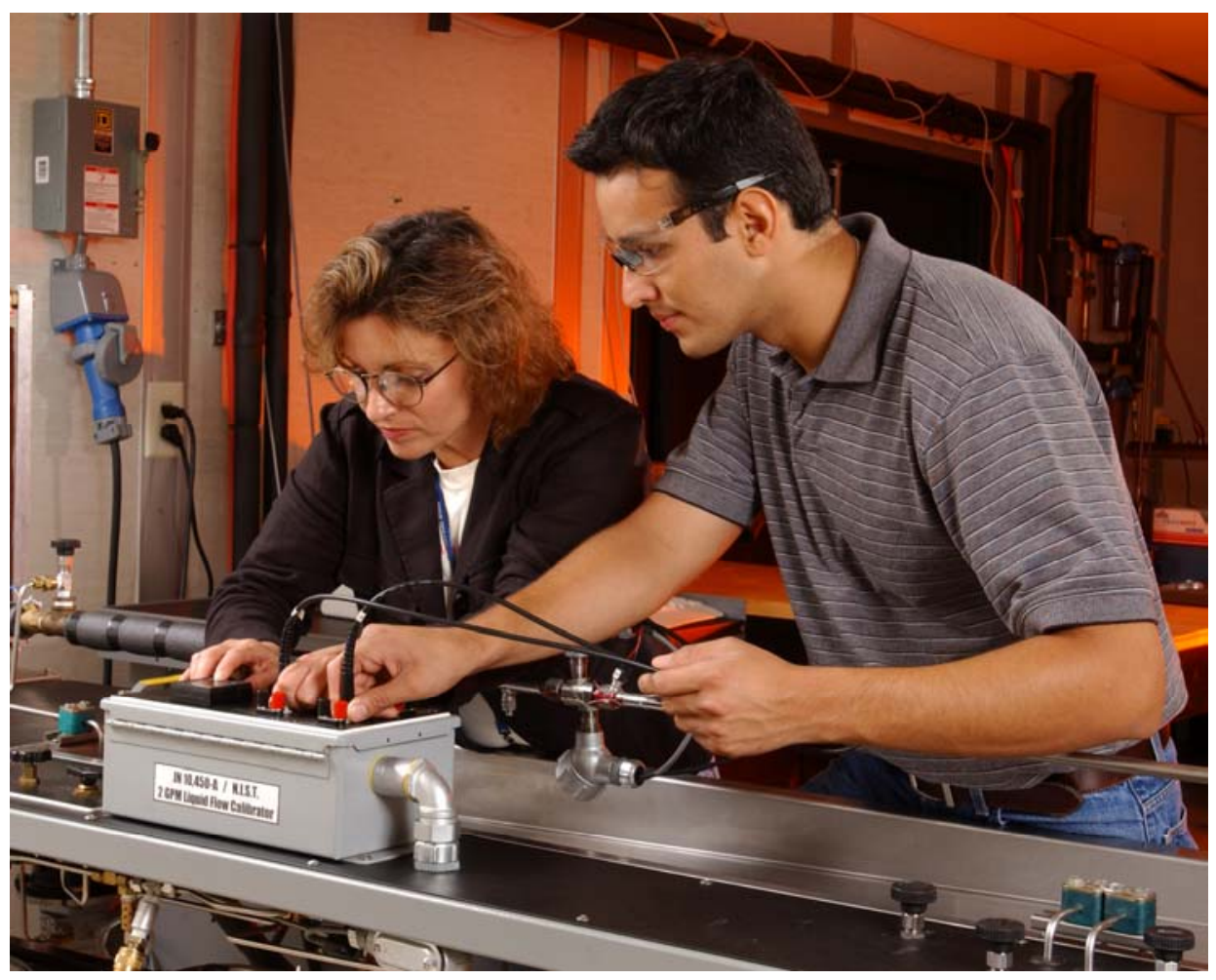

T. T. Yeh, Jesús Aguilera, and John D. Wright

Fluid Metrology Group

Process Measurements Division

Chemical Science and Technology Laboratory

National Institute of Standards and Technology

Gaithersburg, MD 20899 


\section{Hydrocarbon Liquid Flow Calibration Service}

\section{Table of Contents}

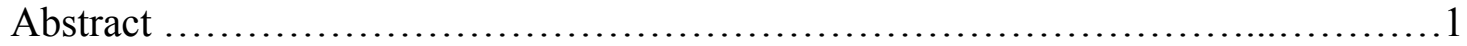

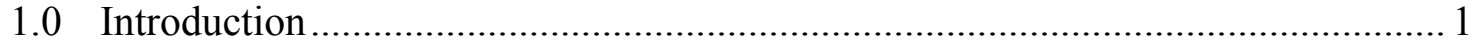

2.0 Description of Measurement Services .......................................................... 2

3.0 Procedures for Submitting a Flowmeter for Calibration...................................... 3

4.0 Description of the Hydrocarbon Liquid Flow Standard ....................................... 4

4.2 The Equation for Flow at the Meter Under Test ........................................... 7

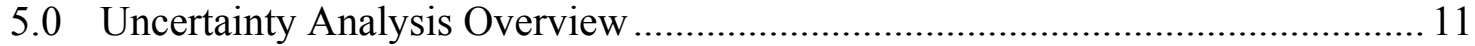

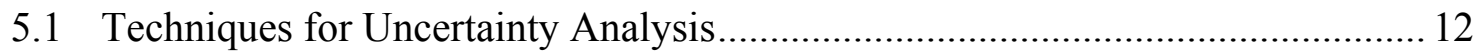

5.2 Measured Quantities and Their Uncertainties................................................. 13

5.3 Propagating Components of Uncertainty ................................................. 19

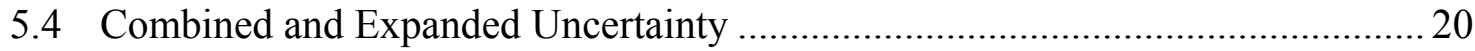

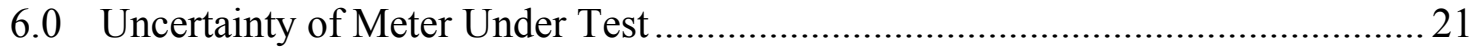

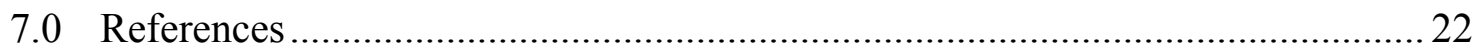

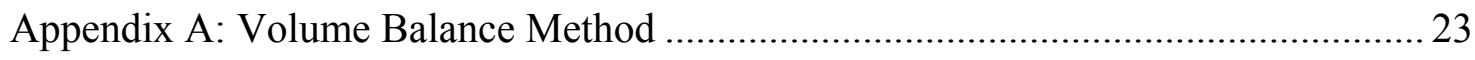

Appendix B: Supporting Data for Uncertainty Analysis ...................................... 28

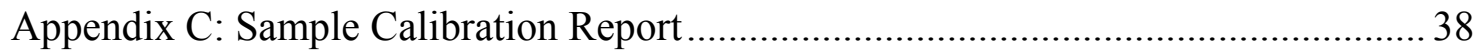




\begin{abstract}
This document provides a description of a 2 liter hydrocarbon liquid flow calibration standard operated by the National Institute of Standards and Technology (NIST) Fluid Metrology Group to provide flowmeter calibrations for customers. This facility uses a passive piston prover technique where fluid is driven by pumps while the measuring piston is pushed by the fluid over a measured length during a measured time. This facility is presently operated using MIL-C7024C fluid, ${ }^{*}$ but other fluids could be used to offer a different range of fluid properties. The facility has uncertainty of $\pm 0.01 \%(k=2$ or approximately $95 \%$ confidence level $)$ over the flow range $0.19 \mathrm{~L} / \mathrm{min}$ to $5.7 \mathrm{~L} / \mathrm{min}(0.05 \mathrm{gal} / \mathrm{min}$ to $1.5 \mathrm{gal} / \mathrm{min})$.

In this document, we provide an overview of the hydrocarbon liquid flow calibration service and the procedures for customers to submit their flowmeters to NIST for calibration. We derive the equation for calculating flow at the meter under test, including the corrections for storage effects caused by changes in fluid density and connecting volume (due to temperature changes). Finally we analyze the uncertainty of the flow standard, give supporting data, and provide a sample calibration report.
\end{abstract}

Key words: calibration, flow, connecting volume, flowmeter, hydrocarbon liquid, flow standard, uncertainty

\title{
1.0 Introduction
}

Flow measurement units are derived from the SI base units. Therefore, the paths taken to realize of flow measurement standards vary and depend upon such issues as the properties of the fluid(s) to be measured. Realization methods at NIST are always derived from fundamental measurements such as mass, length, time, and temperature, typically by accounting for the transfer of a known mass or volume of fluid over a measured time interval under approximately steady state conditions of flow, pressure, and temperature at the meter under test (MUT). Such flow metrology facilities are known as "primary flow standards", and by definition [1], they are facilities capable of determining flow, at specific, quantifiable uncertainty levels, without being calibrated for the unit of flow being realized.

For many years, NIST has provided flowmeter calibrations in hydrocarbon liquid flows up to $1500 \mathrm{~L} / \mathrm{min}$ using a dynamic gravimetric flow standard that has uncertainty of $0.12 \%$. Recently, we have worked to replace the gravimetric standard with new, lower uncertainty facilities that also have a higher level of automation. This document describes the first of these new systems, a 2 liter Hydrocarbon Liquid Flow Standard that covers flows up to $5.7 \mathrm{~L} / \mathrm{min}$ with uncertainty of $0.01 \%$.

The 2 liter Hydrocarbon Liquid Flow Standard (2 L HLFS) consists of a fluid source (e.g., tank), a long test section that provides stable thermodynamic conditions and a fully developed flow profile, and a system for timing the displacement of a quantity of the fluid. The flow measured by the primary standard is computed along with the average of the flow indicated by the MUT during the collection interval. All of the quantities measured in connection with the calibration standard (i.e., temperature, pressure, density, time, etc.) are traceable to established national standards.

\footnotetext{
* Also know as Stoddard solvent - a surrogate liquid for JP-4 and JP-5 jet fuels.
} 
NIST calibrates hydrocarbon liquid flowmeters to provide traceability for flowmeter manufacturers, secondary flow calibration laboratories, and flowmeter users. We calibrate a customer's flowmeter and deliver a calibration report that documents the calibration procedure, the calibration results, and their uncertainty on a fee-for-service basis. The customer may use the flowmeter and its calibration results in different ways. The flowmeter is often used as a transfer standard to compare the customer's primary standards to the NIST primary standards so that the customer can establish traceability, validate their uncertainty analysis, and demonstrate proficiency. Customers with no primary standards use their NIST calibrated flowmeters as working standards or reference standards in their laboratory to calibrate other flowmeters. The Report of Calibration is the property of the customer and NIST considers the results of calibrations to be proprietary information of the customer.

Operators of primary flow standards seek to validate the claimed uncertainties of their standards by establishing and maintaining the traceability of calibration results to the SI. One complete way to establish traceability involves the use of proficiency testing techniques, which quantify the traceability of a facility's results using a set of flow standards maintained by a National Metrology Institute (NMI) [2]. Alternatively, establishing traceability can be achieved through assessment of individual facility components and analyzing their respective contributions to the calibration process. An extensive component analysis of NIST's HLFS has been performed and is described in following sections of this document using the component analysis method.

\subsection{Description of Measurement Services}

Customers should consult the web address www.nist.gov/fluid flow to find the most current information regarding our calibration services, calibration fees, technical contacts, and flowmeter submittal procedures.

NIST uses the hydrocarbon liquids standards described herein to provide hydrocarbon liquids flowmeter calibrations for flows between $0.19 \mathrm{~L} / \mathrm{min}$ and $5.70 \mathrm{~L} / \mathrm{min}$ [3]. The facility can be used at flows as low as $0.05 \mathrm{~L} / \mathrm{min}$ or as high as $7 \mathrm{~L} / \mathrm{min}$, but calibrations below $0.19 \mathrm{~L} / \mathrm{min}$ or over $5.7 \mathrm{~L} / \mathrm{min}$ should be discussed with the technical contacts before a flowmeter is submitted for such calibrations.

The liquid used for calibrations is normally MIL-C-7024C fluid (also known as Stoddard or Type 2 solvent), but calibrations using other fluids are possible. The hydrocarbon liquid temperature during the calibration is nominally $22.2 \pm 0.5^{\circ} \mathrm{C}$. Readily available fittings for the installation of flowmeters for calibration are Swagelok ${ }^{*}, \mathrm{~A} / \mathrm{N} 37$ degree flare, and national pipe thread (NPT).

Meters are tested if the flow range and piping connections are suitable, and have precision appropriate for calibration with the NIST flow measurement uncertainty. The vast majority of flowmeters calibrated in the hydrocarbon liquids flow calibration service are turbine flowmeters because these are presently regarded as the best candidates for transfer and working standards by the hydrocarbon liquid flow metrology community. Meter types with calibration instability significantly larger than the primary standard uncertainty are generally not calibrated with the

\footnotetext{
* Certain commercial equipment, instruments, or materials are identified in this paper to foster understanding. Such identification does not imply recommendation or endorsement by the National Institute of Standards and Technology, nor does it imply that the materials or equipment identified are necessarily the best available for the purpose.
} 
NIST standards since the job can be done with acceptable uncertainty more economically by other, commercial labs.

A normal flow calibration performed by the NIST Fluid Metrology Group consists of six flows spread over the range of the flowmeter. A flowmeter is normally calibrated at $10 \%, 20 \%, 35 \%$, $50 \%, 75 \%$, and $100 \%$ of its full scale. At each of these flow set points, five (or more) flow measurements are made consecutively. The same set point flows are tested on a second occasion, but the flows are tested in decreasing order instead of the increasing order of the first set. Therefore, the final data set consists of ten (or more) flow measurements made at six flow set points, i.e., 60 individual flow measurements. The sets of five measurements can be used to assess repeatability, while the sets of ten can be used to assess reproducibility. For further explanation, see the sample calibration report in Appendix $C$ of this document. Variations on the number of flow set points, spacing of the set points, and the number of repeated measurements can be discussed with the NIST technical contacts. However, for data quality assurance reasons, we rarely will conduct calibrations involving fewer than three flow set points and two sets of three flow measurements at each set point.

The Fluid Metrology Group prefers to present flowmeter calibration results in a dimensionless format that takes into account the physical model for the flowmeter type. The dimensionless approach facilitates accurate flow measurements by the flowmeter user even when the conditions of usage (liquid type, temperature) differ from the conditions during calibration. Hence for a turbine calibration, the calibration report will present Roshko number vs Strouhal number [4]. In order to calculate the uncertainty of these flowmeter calibration factors, we must know the uncertainty of the standard flow measurement as well as the uncertainty of the instrumentation associated with the MUT (normally frequency and temperature instrumentation). NIST-owned and controlled instruments (temperature, etc.) are used as part of the test of a customer's meter since these have established uncertainty values based on calibration records maintained as part of the our Quality System. Such information is not available for the customer's instrumentation. Use of the customer's instrumentation for a calibration or special test requires specific arrangements to be made for such operations. Calibration of customer's ancillary instrumentation is not part of the calibration procedures described here and would require that separate arrangements be made.

\subsection{Procedures for Submitting a Flowmeter for Calibration}

The Fluid Metrology Group follows the policies and procedures described in Chapters 1, 2, and 3 of the NIST Calibration Services Users Guide. These chapters can be found on the internet at the following addresses:

http://ts.nist.gov/ts/htdocs/230/233/calibrations/Policies/policy.htm, http:/ts.nist.gov/ts/htdocs/230/233/calibrations/Policies/domestic.htm, and http://ts.nist.gov/ts/htdocs/230/233/calibrations/Policies/foreign.htm.

Chapter 2 gives instructions for ordering a calibration for domestic customers and has the subheadings: A.) Customer Inquiries, B.) Pre-arrangements and Scheduling, C.) Purchase Orders, D.) Shipping, Insurance, and Risk of Loss, E.) Turnaround Time, and F.) Customer Checklist. Chapter 3 gives special instructions for foreign customers. The web address

www.nist.gov/fluid flow has information more specific to the flow calibration service, including the technical contacts in the Fluid Metrology Group, fee estimates, and turn around times. 


\subsection{Description of the Hydrocarbon Liquid Flow Standard}

Piston prover systems have long been accepted as primary flow calibrators for both gas and liquid flowmeters $[5,6]$. In its most basic form, the piston prover consists of a circular cylinder of known internal diameter, which encompasses a sealed piston. This piston strokes through measured lengths, at a constant speed, to produce a volumetric flow. The cross sectional area of the cylinder multiplied by the length traveled gives a volume. The volumetric flow is calculated by dividing the volume by the time it took the piston to traverse the length. Temperature measurements at key locations are used to assess the changes in fluid density and connecting volume that occur between the start and stop times, allowing corrections for storage effects. The coefficient of thermal expansion of the calibration fluid is known, hence the volumetric flow in the cylinder can be converted to the volumetric flow at the test section where the meter under test (MUT) is located (or the mass flow can be determined from the fluid density). The output of the meter under test is acquired along with the necessary piston measurements so that average flows from the MUT and the flow standard can be compared.

Two types of piston arrangements are generally used in these systems. An active piston can both drive and measure a volumetric flow out of the cylinder (like a syringe), while a passive piston operates via pumped fluid, which drives the piston through the calibration stroke to determine the volumetric flow. The NIST HLFS employs a passive piston.

Table 1. Nominal Characteristics of NIST's 2 liter Hydrocarbon Liquid Flow Standard.

$\begin{array}{cc}\Delta V_{p},\left[\mathrm{~cm}^{3}\right] & 560 \text { to } 1530^{* *} \\ D,[\mathrm{~cm}] & 7.62 \\ d,[\mathrm{~cm}] & 2.54 \\ L,[\mathrm{~cm}] & 55 \\ L_{c},[\mathrm{~cm}] & 14 \text { to } 40^{* *} \\ t_{c},[\mathrm{~s}] & 15 \text { to } 180^{* *} \\ u_{C},[\%] & 0.005 \\ u_{k=2},[\%] & 0.01 \\ Q,[\mathrm{~L} / \mathrm{min}] & 0.19 \text { to } 5.7\end{array}$

${ }^{* *}$ Specific values are selected to conform with those used in the following uncertainty analyses. 


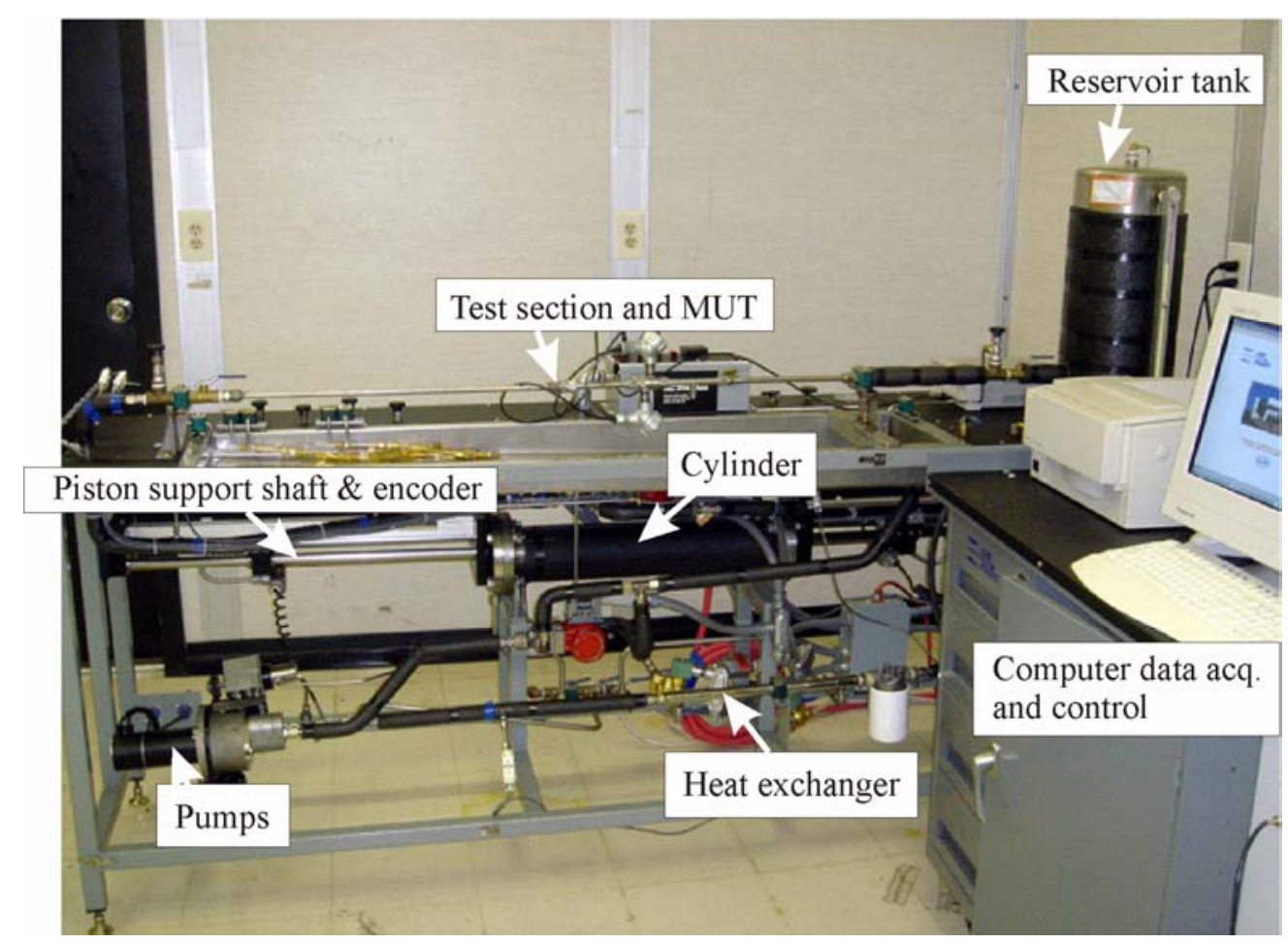

Figure 1. Photograph of the small Liquid Flow Calibrator.

The 2 L HLFS was constructed by Flow Dynamics Inc, in Scottsdale, AZ. ${ }^{*}$ Upon receipt at NIST, we performed the calibrations of length, time, temperature, and fluid properties necessary to make the system directly traceable to NIST standards and made an uncertainty analysis and those efforts are documented herein.

The HLFS is operated in a closed loop mode. As shown schematically in Figure 2, the hydrocarbon liquid is pumped from the reservoir tank by two pumps. Depending on the required flow, some of the pumped liquid is returned to the reservoir directly. The remaining, required flow is passing through the piston-valve assembly and then to the test section, where the MUT is located. After the MUT, the entire flow is returned to the reservoir to complete the flow loop.

The HLFS uses two three-way valves to allow the pumped liquid to move the piston back and forth in a bi-directional mode while maintaining unidirectional flow through the MUT. Calibration data is acquired with the piston moving in either direction.

* Certain commercial equipment, instruments, or materials are identified in this paper to foster understanding. Such identification does not imply recommendation or endorsement by the National Institute of Standards and Technology, nor does it imply that the materials or equipment identified are necessarily the best available for the purpose. 


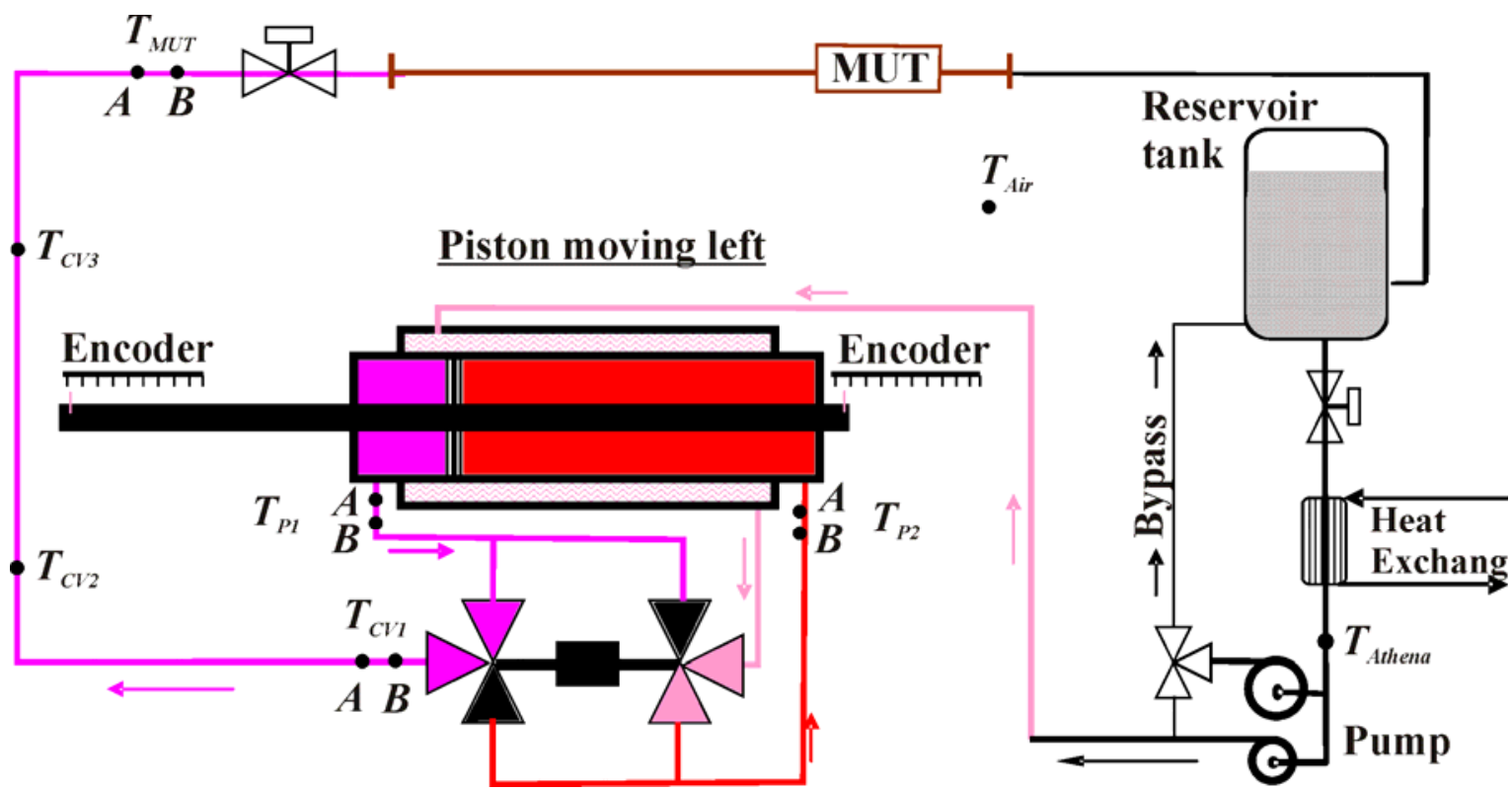

Figure 2. A sketch of the calibrator with the piston stroking left. The locations of temperature sensors are shown.

The operation of the four-way diverter valve is demonstrated schematically in Figures 2 and 3. The calibration interval begins as soon as flow conditions reach steady state and the piston accelerates to constant velocity (shortly after the piston begins travel in one direction). During the transition period when the piston changes direction, both of the three-way valves are set so that all three ports are open, thus preventing any hydraulic ram effects. During the change in direction, flow stops at the MUT, and it is necessary to wait for steady state conditions before beginning to collect calibration data. The meter output averaging is stopped before the piston reaches the transition period.

To allow a range of flows, a computer-controlled stepping-motor drives both pumps. The capacity of the small pump is about one tenth the larger one. At lower flow conditions, the flow from the large pump is bypassed to the reservoir tank.

Steady state temperature conditions are achieved by cycling the piston back and forth at the intended calibration flow, which promotes the mixing of the fluid. A chilled water heat exchanger controlled by a feedback temperature sensor is used to remove heat from the fluid added by friction and the pumps. Thermal equilibrium is further enhanced by forcing the calibration fluid through an outer cylindrical jacket that encloses the cylinder and piston. The liquid flow from the pumps is directed into this outer jacket before entering the four-way diverter valve. Two benefits of using this outer jacket are: (1) better temperature uniformity among different parts of the fluid and the prover, and (2) pressure balance inside and outside of the prover, preventing a pressure gradient from affecting the diameter of the cylinder. The calibrator uses twelve temperature sensors to determine fluid properties throughout the flow loop. The locations are indicated in Figure 2. Furthermore, the calibrator is located in a temperaturecontrolled room where the air temperature is maintained at $22.2 \pm 1^{\circ} \mathrm{C}$. These considerations are 
important for minimizing changes in density in the connecting volume between the cylinder and the MUT and the resulting corrections for storage effects.

(a) Piston Stroking Left

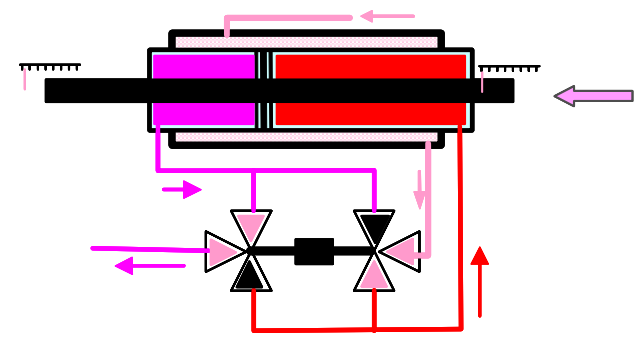

(c) Piston Stroking Right

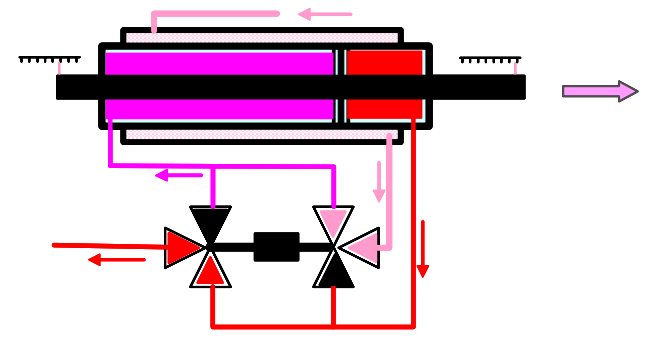

(b) Transition from Left to Right Stroke

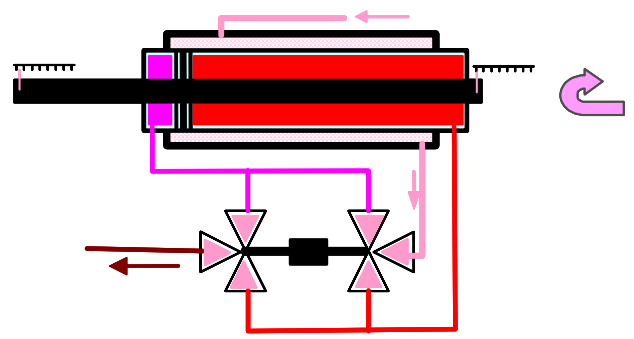

(d) Transition from Right to Left stroke

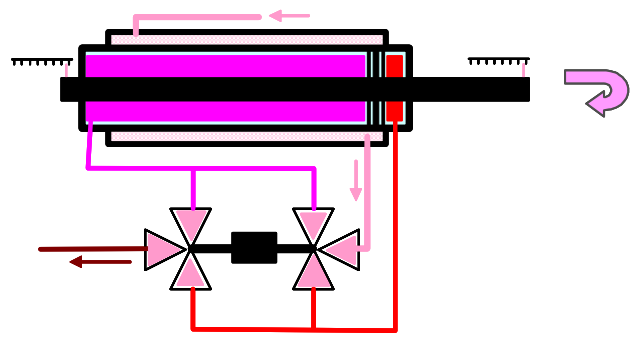

Figure 3. Diagrams of piston and three-way valves reversing piston directions.

The position of the piston along the cylinder length is measured with two, redundant linear encoders, one on each of the supporting shafts attached to either side of the piston. The encoders output nominally 50 square wave pulses per $\mathrm{mm}$ of length traveled. The time for the piston to travel a given length is measured by counting pulses from two redundant $1 \mathrm{MHz}$ timers (see section 5.2).

Leaks of calibration fluid can be visually detected. There are two o-ring seals on the piston, one on the leading edge, another on the trailing edge. Fluid that leaks past either of these seals flows through machined holes in the piston and piston shafts to the shaft ends where it will drip out. Leaks past the piston shaft seals and from pipe fittings are easy to detect by eye.

\subsection{The Equation for Flow at the Meter Under Test}

The HLFS measures the volumetric flow of fluid through the MUT from the fluid discharged by the piston-cylinder assembly over a precisely measured time period. Since leaks or temperature and pressure changes may cause the total mass in the pipe that connects the piston-cylinder assembly to the MUT (the connecting volume) to change during the calibration, the flow through this meter may differ from the flow exiting the cylinder (storage effects). If no leaks are 
found, if corrections for temperature and pressure effects have been made, and if the fluid volume change in the connecting pipe is accounted for, the volumetric flow at the MUT can be obtained. Most previous prover analyses do not include corrections for fluid mass change in the connecting volume and fluid density is assumed constant during the calibration (they are treated as uncertainties instead). However, the connecting volume effect could contribute a significant error in the volumetric flow determination.

Different methods can be used in accounting for the connecting volume effect. The most common correction technique, which is based on the mass conservation method, is given below. A different method based on volume balance is given in Appendix A.

Conservation of Mass

The operational principle of the piston prover is the conservation of mass. The general equation for the conservation of mass is:

$$
\frac{\partial}{\partial t} \int_{V} \rho d V+\int_{A} \rho \vec{U} \cdot d \vec{A}=0
$$

where $\rho$ is the fluid density; $\partial / \partial t$ is the partial derivative with respect to time; $V$ is a control volume used to assess the specified mass balance; and $\vec{U}$ is the vectorial velocity across the vectorial area element, $d \vec{A}$, of the control surface (surrounding the control volume) with the positive direction outward, such that the positive $\vec{U} \cdot d \vec{A}$ product denotes outflow. Equation (1) states that mass is neither created nor destroyed. For this system, that is, the initial fluid mass in the control volume, at the beginning of the calibration time interval, equals the final fluid mass at the end of the calibration,

$$
M_{F i}=M_{F f}
$$

where the subscripts $i$ and $f$ denote the initial and final conditions. For a piston prover calibrator as shown in Figure 4, the initial fluid mass $M_{F i}$ is

$$
M_{F i}=M_{P i}+M_{C V i}+M_{M U T i}
$$

and the final fluid mass $M_{F f}$ is

$$
M_{F f}=M_{P f}+M_{C V f}+M_{M U T f}+M_{L}
$$

In (3) and (4), the subscripts $P, C V$, and $M U T$ denote the piston, connecting volume, and MUT, respectively. $M_{L}$ is any mass leaking from the system between the prover and MUT. The leakage is usually apparent and leaks are repaired before the calibrator can be used. Therefore, the leakage term $M_{L}$ is hereafter assumed to be zero. 


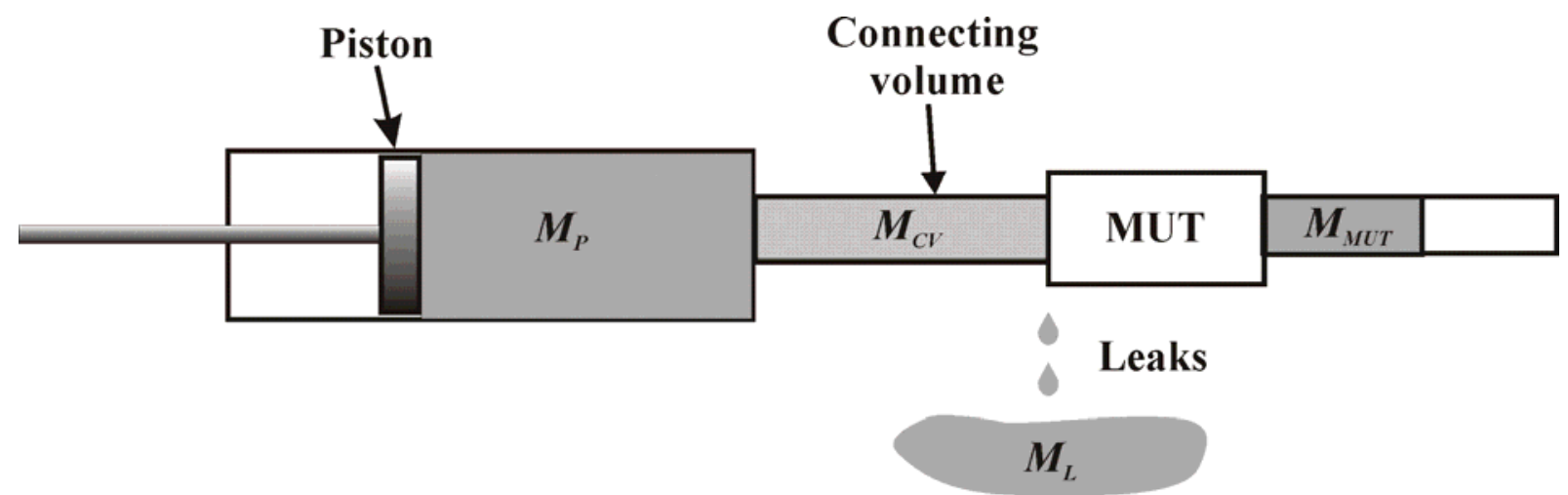

Figure 4. Mass conservation as applied to an incompressible piston system.

From (2) (4), we have the mass of fluid passing through the MUT as:

$$
\Delta M_{M U T}=M_{M U T f}-M_{M U T i}=\Delta M_{P}-\Delta M_{C V}
$$

where

$$
\Delta M_{P}=M_{P i}-M_{P f}=\int_{V_{P i}} \rho d V-\int_{V_{P f}} \rho d V
$$

is the total mass displaced by the piston during the time period, $t_{c}$, and

$$
\Delta M_{C V}=M_{C V f}-M_{C V i}
$$

is the total mass difference in the connecting volume during the same period, $t_{c}$. When the fluid density in the piston is constant during the calibration time and equal to $\rho_{P},(6)$ simplifies to:

$$
\Delta M_{P}=\int_{V_{P i}} \rho d V-\int_{V_{P f}} \rho d V=\rho_{P i} V_{P i}-\rho_{P f} V_{P f}=\rho_{P} \Delta V_{P}
$$

where $\Delta V_{P}$ is the total volume displaced by the piston moving along the cylinder in the calibration in time, $t_{c}$.

\section{Connecting Volume Effects}

In general, the fluid density is a function of pressure and temperature. However, in this liquid calibrator, the effect of the pressure variation is negligible, since the pressure is nearly constant throughout the system, the maximum operating pressure is $200 \mathrm{kPa}$, and the modulus of elasticity of the calibration liquid is large $(\simeq 2 \mathrm{GPa})$. Therefore, in this analysis, the liquid density is assumed to be a function of the temperature only. Similarly, the structural length and volume of the connecting piping is assumed to be a function of temperature only (i.e., no 
structural deformation in the pipe due to liquid pressure). For a small temperature change, $\Delta T$, the following linear relationships are assumed:

$$
\begin{aligned}
\rho & =\rho_{0}(1-\alpha \Delta T) \\
L & =L_{0}\left(1+\alpha_{s} \Delta T\right) \\
V & =V_{0}\left(1+3 \alpha_{s} \Delta T\right)
\end{aligned}
$$

where $\alpha$ is the thermal expansion coefficient for the liquid density, and $\alpha_{s}$ is the linear expansion coefficient for the connecting pipe. Thus, any mass change in the connecting volume can be expressed as:

$$
\begin{array}{rcc}
\Delta M_{C V}= & \Delta(\rho V)_{C V} \\
= & \rho_{C V} \Delta V_{C V}+V_{C V} \Delta \rho_{C V} \\
= & \rho_{C V}\left(V_{C V} 3 \alpha_{s} \Delta T_{C P}\right)-V_{C V}\left(\rho_{C V} \alpha \Delta T_{C V}\right) \\
= & \left(\rho_{C V} V_{C V}\right)\left(3 \alpha_{S} \Delta T_{C P}-\alpha \Delta T_{C V}\right)
\end{array}
$$

where $\rho_{C V}$ is the average fluid density in the connecting volume; $V_{C V}$ is the average connecting volume; $\Delta T_{C P}$ is the temperature rise of the connecting pipe between the initial and final times for the calibration interval; and $\Delta T_{C V}$ is the temperature rise of the fluid in the connecting volume between the initial and final times.

Substituting (8) and (10) into (5), the fluid mass through the MUT in the calibration interval, $t_{c}$, becomes

$$
\Delta M_{M U T}=\rho_{P} \Delta V_{P}-\left(\rho_{C V} V_{C V}\right)\left(3 \alpha_{s} \Delta T_{C P}-\alpha \Delta T_{C V}\right)
$$

Dividing (11) by the fluid density in the MUT, $\rho_{M U T}$, and the calibration interval, $t_{c}$, the average volumetric flow rate through the MUT, $Q_{M U T}$, becomes:

$$
Q_{M U T}=\frac{\Delta M_{M U T}}{\rho_{M U T} t_{c}}=\frac{\rho_{P} \Delta V_{P}-\left(\rho_{C V} V_{C V}\right)\left(3 \alpha_{s} \Delta T_{C P}-\alpha \Delta T_{C V}\right)}{\rho_{M U T} t_{c}}
$$

From (9), the density ratios can be expressed as:

$$
\frac{\rho_{P}}{\rho_{M U T}}=1-\alpha \Delta T_{P, M U T} \text { and } \frac{\rho_{C V}}{\rho_{M U T}}=1-\alpha \Delta T_{C V, M U T}
$$

where $\Delta T_{P, M U T}$ is the temperature difference between the fluid in the piston prover and the fluid in the MUT and $\Delta T_{C V, M U T}$ is the average temperature difference between the fluid in the 
connecting volume and that in the MUT. Substituting equation (13) into (12), the volumetric flow rate equation becomes:

$$
Q_{M U T}=\frac{1}{t_{c}}\left[\begin{array}{l}
\Delta V_{P}\left(1-\alpha \Delta T_{P, M U T}\right)-V_{C V}\left(3 \alpha_{S} \Delta T_{C P}-\alpha \Delta T_{C V}\right) \\
+\alpha \Delta T_{C V, M U T} V_{C V}\left(3 \alpha_{S} \Delta T_{C P}-\alpha \Delta T_{C V}\right)
\end{array}\right]
$$

The last term in (14) is a second order expansion quantity which can be neglected based on a magnitude analysis. Hence, the final equation for volumetric flow rate becomes:

$$
Q_{M U T}=\frac{1}{t_{c}}\left[\Delta V_{P}\left(1-\alpha \Delta T_{P, M U T}\right)+V_{C V}\left(\alpha \Delta T_{C V}-3 \alpha_{S} \Delta T_{C P}\right)\right]
$$

Equation (15) is the basis equation for calculating flow from the HLFS. The first term in the parentheses represents the flow from the piston volume with a thermal expansion correction. The second term in the parentheses accounts for storage effects in the connecting volume, 1) due to density changes in the fluid caused by temperature changes and 2) due to changes in the volume of the tubing holding the fluid.

From (15), it can be seen that the fluid density does not directly affect the calculation of the volumetric flow rate; however, any change in fluid density in the connecting volume is given by the terms $-\alpha \Delta T_{P, M U T}$ and $V_{C V} \alpha \Delta T_{C V}$. Thus the value of liquid density is only important if a mass flow, $\rho_{M U T} Q_{M U T}$, is needed.

An alternate method, using volume balance, for correcting fluid volume in the connecting volume gives a same result as that of the equation (15). The volume balance method is given in Appendix A.

\subsection{Uncertainty Analysis Overview}

The uncertainty components of the Hydrocarbon Liquid Flow Standard are discussed in detail in following sections. As seen in (15), they include the uncertainty of the following elements:

a. the displaced prover volume, $\Delta V_{P}$, and the linear thermal expansion coefficient for the piping, $\alpha_{S}$,

b. several temperature differences $\left(\Delta T_{P, M U T}, \Delta T_{C P}, \Delta T_{C V}\right)$,

c. the connecting volume, $V_{C V}$,

d. the calibration interval, $t_{c}$, and

e. the thermal expansion coefficient of the liquid density, $\alpha$.

Some component uncertainties listed above and in Equation (15) could not be measured directly. Their uncertainties are estimated from the uncertainties of their source measurements using the first order uncertainty propagation method to be discussed below. Figure 5 shows a graphic representation tree of the uncertainty analysis for the HLFS. 


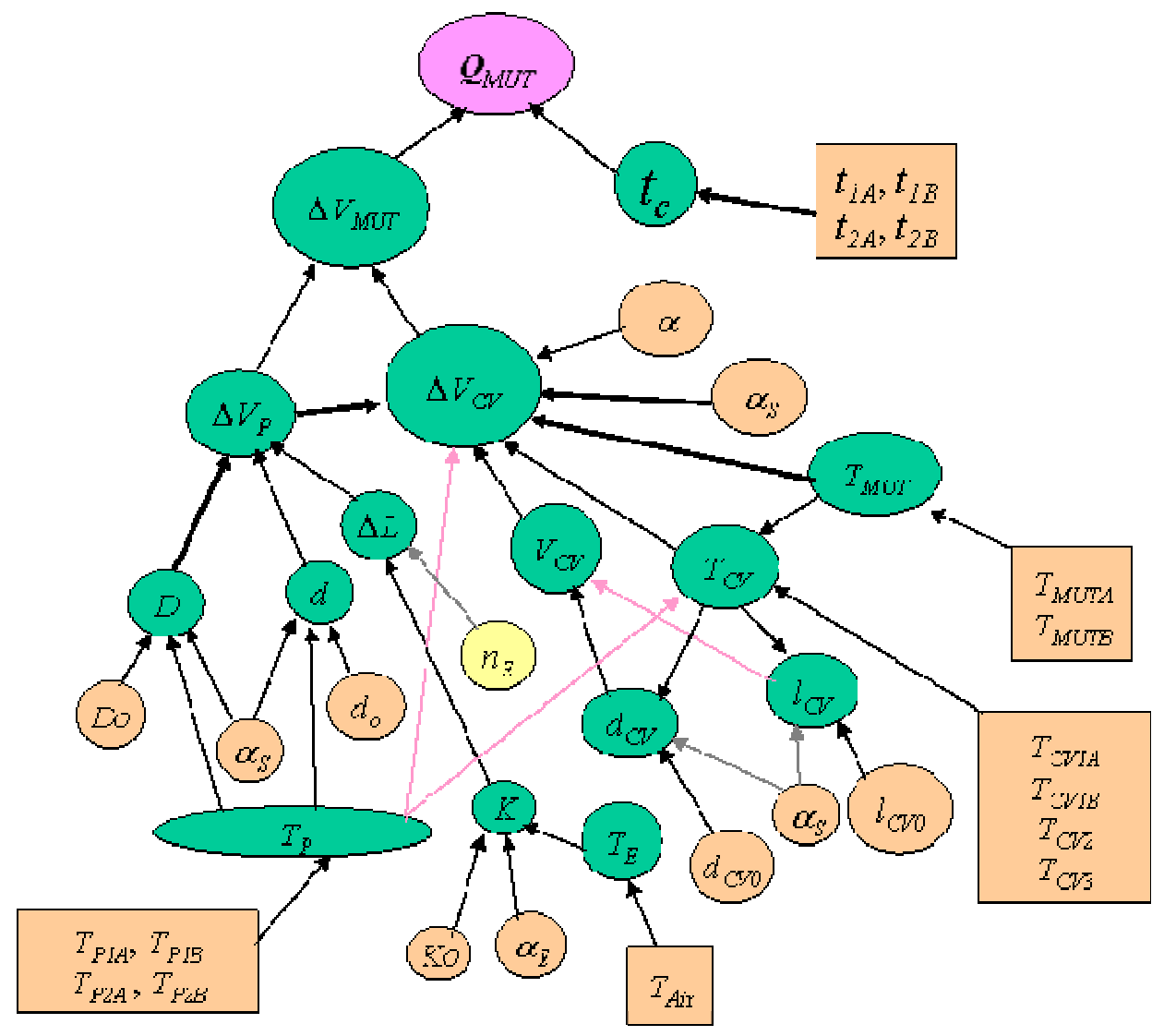

'Figure 5. Graphic representation of the uncertainty analysis.

\subsection{Techniques for Uncertainty Analysis}

Here we follow the guidelines for evaluating and expressing uncertainty provided in NIST TN 1297 [7], the ISO Guide [8], and elsewhere [9]. In general, if a measurement quantity, $y$, is a function of variables $x_{i}$,

$$
y=f\left(x_{1}, x_{2}, \ldots \ldots, x_{n}\right)
$$

its first-order Taylor series approximation is,

$$
d y=\sum_{i} \frac{\partial y}{\partial x_{i}} d x_{i}
$$

Thus, the propagation of uncertainty yields 


$$
\begin{aligned}
u_{c}^{2}(y) & =\left[\sum_{i=1}^{n}\left(\frac{\partial y}{\partial x_{i}}\right) u\left(x_{i}\right)\right]^{2} \\
& =\sum_{i=1}^{n}\left(\frac{\partial y}{\partial x_{i}}\right)^{2} u^{2}\left(x_{i}\right)+2 \sum_{i=1}^{n-1}\left(\frac{\partial y}{\partial x_{i}}\right) \sum_{j=i+1}^{n}\left(\frac{\partial y}{\partial x_{j}}\right) r_{i j} u\left(x_{i}\right) u\left(x_{j}\right)
\end{aligned}
$$

where $u_{c}(y)$ is the combined standard uncertainty of the measurement result $y, u\left(x_{i}\right)$ is the standard uncertainty of the variable $x_{i}$, the partial derivatives $\partial y / \partial x_{i}$ are the dimensional sensitivity coefficients of $x_{i}$ on $y$, and $r_{i j}$ is the cross correlation coefficient between variables $x_{i}$ and $x_{j}$. An alternative form of (18), which expresses the uncertainty propagation in a dimensionless form, is shown below and it is often more useful.

$$
u_{y}^{2}=\sum_{i=1}^{n} c_{x_{i}, y}^{2} u_{x_{i}}^{2}+2 \sum_{i=1}^{n-1} c_{x_{i}, y} \sum_{j=i+1}^{n} c_{x_{j}, y} r_{i j} u_{x_{i}} u_{x_{j}}
$$

In (19), $u_{y}=u_{c}(y) / y$ is the combined dimensionless standard uncertainty of the measurement result $y, c_{x_{i}, y}=\left(\partial y / \partial x_{i}\right) x_{i} / y$ are the dimensionless sensitivity coefficients of $x_{i}$ on $y$, and $u_{x_{i}}=u\left(x_{i}\right) / x_{i}$ is the dimensionless uncertainty of the variable $x_{i}$. Equation (19) is used here to estimate the combined uncertainty of the measurement. In many cases, the uncertainty of $x_{i}$ could not be measured directly. For those cases, the same uncertainty propagation given by (19) is used for a sub-measurement process to estimate the combined uncertainty of the sub-measurement. This process is propagated throughout all the measurement components needed until the desired measured quantities are obtained.

According to [8], the sources of uncertainty used in assessing the combined standard uncertainty of the measurement process can be classified according to two types: Type $A$ - those which are evaluated by statistical methods, and Type $B$ - those which are evaluated by other means. Following this convention, each measured quantity has been classified accordingly as a $u_{A}$ or $u_{B}$.

\subsection{Measured Quantities and Their Uncertainties}

As noted above, the uncertainty propagation can be calculated based on (15), but it is worth remembering that the flow and the related components in (15) are not directly measured. To obtain the uncertainty of this result, its relationship to other measured quantities is required. In this section, the relationships are given.

\section{The Displaced Volume}

The piston prover volume, $\Delta V_{P}$, can be calibrated by: (a) water draw method, (b) using a master flowmeter, or (c) by using the dimensions of its diameter and the distance traveled by the piston. Here, the diameter/traveled-distance method is used to determine the prover volume. The piston displacement is measured using optical encoders. In terms of the measured quantities, $\Delta V_{P}$ is, 


$$
\Delta V_{P}=\frac{\pi\left(D^{2}-d^{2}\right)}{4} \Delta L_{E}=\frac{\pi\left(D^{2}-d^{2}\right)}{4} \frac{n_{E}}{K_{E}}
$$

where $D$ and $d$ are the piston prover diameter and piston shaft diameter, respectively; $\Delta L_{E}$ is the encoder (or piston) displacement length, $K_{E}$ is the encoder constant (in pulses $/ \mathrm{cm}$ ), and $n_{E}$ is the total number of pulses produced by the encoder during the calibration stroke. In our system, the value $n_{E}$ may be selected by software or by the operator, to ensure that the prover is operating at the uncertainty asserted in this analysis. The prover unit was disassembled so the shaft and the cylinder diameters could be measured accurately. The shaft diameter was measured using an outside diameter micrometer (Mitotoyo Model MDC-2"JT). Two standard blocks, one slightly smaller and one slightly larger than the shaft diameter, calibrated by the NIST Precision Engineering Division were used to calibrate the micrometer and establish traceability. Table B3 in Appendix B shows the measurement results and their uncertainties. The cylinder inside diameter was measured using a three-legged inside diameter micrometer (Mitutoyo, Model IT005D). Two standard rings, one slightly smaller and one slightly larger than the cylinder inside diameter, calibrated by the NIST Precision Engineering Division, were used to calibrate the inside micrometer. Table B4 in Appendix B shows the data for the inside diameter and their uncertainties.

In bi-directional piston prover calibrators, the piston diameter is the same, regardless of the direction of piston travel. However, the shafts that are attached to both sides of the piston may have slightly different diameters. Similarly, if multiple linear encoders, as is the case here, monitor the displacement of the piston shafts, they may have slightly different encoder constants. Therefore, for bi-directional provers, the measurement characteristics in one direction of piston travel may be different from the other. Nonetheless, because the shaft diameter assessment process is based on the entire shaft on both sides and the times measured from both encoders are used for either direction (see time measurement below), it is expected that the uncertainty for each direction will be the same.

In this system, the encoders are Mitutoyo AT2N-600. A stabilizer is built into the detector to ensure a stable signal output. The encoder detector head is trued to the glass scale inside its housing and fixed by positioning plates. The thermal expansion of the encoders is given by,

$$
L_{E}=L_{E R}\left(1+\alpha_{E} \Delta T_{E R}\right) \text { and } K_{E}=K_{E R}\left(1-\alpha_{E} \Delta T_{E R}\right)
$$

where $L_{E R}$ and $K_{E R}$ are the encoder length and encoder constant at the reference temperature, respectively; $\Delta T_{E R}$ is the temperature rise from the reference temperature, and $\alpha_{E}$ is the linear expansion coefficient of the encoder. The encoder constant was calibrated using a reference laser interferometer (HP Model 10565A) borrowed from the Precision Engineering Division. The calibration was made by comparing the laser interferometer output with the inline output of the encoder pulses. Table B5 in Appendix B shows the measurement results and their uncertainties.

The diameters of the prover assembly also change with the temperature following these relations,

$$
D=D_{R}\left(1+\alpha_{S} \Delta T_{D R}\right) \text { and } d=d_{R}\left(1+\alpha_{S} \Delta T_{d R}\right)
$$


where $D_{R}$ and $d_{R}$ are the reference temperature diameters of the prover and of the piston shaft, respectively (see Tables $\mathrm{B} 3$ and $\mathrm{B} 4$ ), and $\Delta T_{D R}$ and $\Delta T_{d R}$ are the temperature rise with respect to their reference temperature, respectively.

\section{Temperature Measurement}

The uncertainty of the temperature measurements made throughout the prover will contribute to the uncertainty of the calibrator. Thermistors are used for all temperature measurements, with twelve of them placed at various locations along the liquid flow path. At locations deemed critical, the system has duplicate sensors to improve measurement accuracy. The locations of the temperature sensors in the system are shown in Figure 2. $T_{\text {Athena }}$ is located near the heat exchanger and is used by the temperature controller; $T_{1 A}$ and $T_{1 B}$ are located at prover exit-1, and $T_{2 A}$ and $T_{2 B}$ are at prover exit-2; $T_{C V 1 A}$ and $T_{C V 1 B}$ are located at the exit of the four-way value, $T_{C V 2}$ and $T_{C V 3}$ are placed along the length of the connecting volume; $T_{M U T A}$ and $T_{M U T B}$ are immediately upstream of the MUT.

The model used for the reduction of the various temperatures in the system affects the uncertainty of the calibrator results. At initial and final conditions, the average connecting volume fluid temperature, $T_{C V}$, is assumed to be the average value of the five temperature readings made along the fluid path.

$$
T_{C V}=\left(T_{P}+T_{C V 1}+T_{C V 2}+T_{C V 3}+T_{M U T}\right) / 5
$$

In the above equation, $T_{P}=\left(T_{P i A}+T_{P i B}\right) / 2$ is the average prover temperature (depending on the piston travel direction, $i=1$ or 2$) ; T_{C V 1}=\left(T_{C V 1 A}+T_{C V 1 B}\right) / 2$ is the average temperature at exit of the four way valve; and $T_{M U T}=\left(T_{M U T A}+T_{M U T B}\right) / 2$ is the temperature at the MUT. This average temperature model results in an uncertainty for the average temperature. This additional uncertainty in the average temperature is given by.

$$
E_{\max }=\text { the maximum spatial variation of the fluid temperature. }
$$

Typical temperature variation in the connecting volume is shown in Figure 6. This and other test data show that the maximum temperature variation among the sensor locations is $0.1 \mathrm{~K}$ or less.

The thermistors are periodically calibrated in an isothermal bath by comparing their response to that of a standard thermometer calibrated by the NIST Thermometry Group. The four calibration coefficients and the temperature uncertainty for each thermistor are obtained using a linear regression method. The uncertainty in the reference temperature, which is $0.002 \mathrm{~K}$, is also classified as a Type B uncertainty for each sensor. An additional uncertainty is obtained by comparing the temperature output of the calibrated sensors with the reference temperature in the isothermal bath. The results from a sample calibration are shown in Tabel B1 in Appendix B. The average difference is assigned as the Type B uncertainty and the standard deviation of the difference is assigned as a Type A uncertainty. The root-sum-square of the uncertainties from the 
reference sensor, data regression, and temperature test, is the combined uncertainty of each sensor. In the worst case, the sensors have $u_{A}=0.041 \mathrm{~K}$ and $u_{B}=0.031 \mathrm{~K}$.

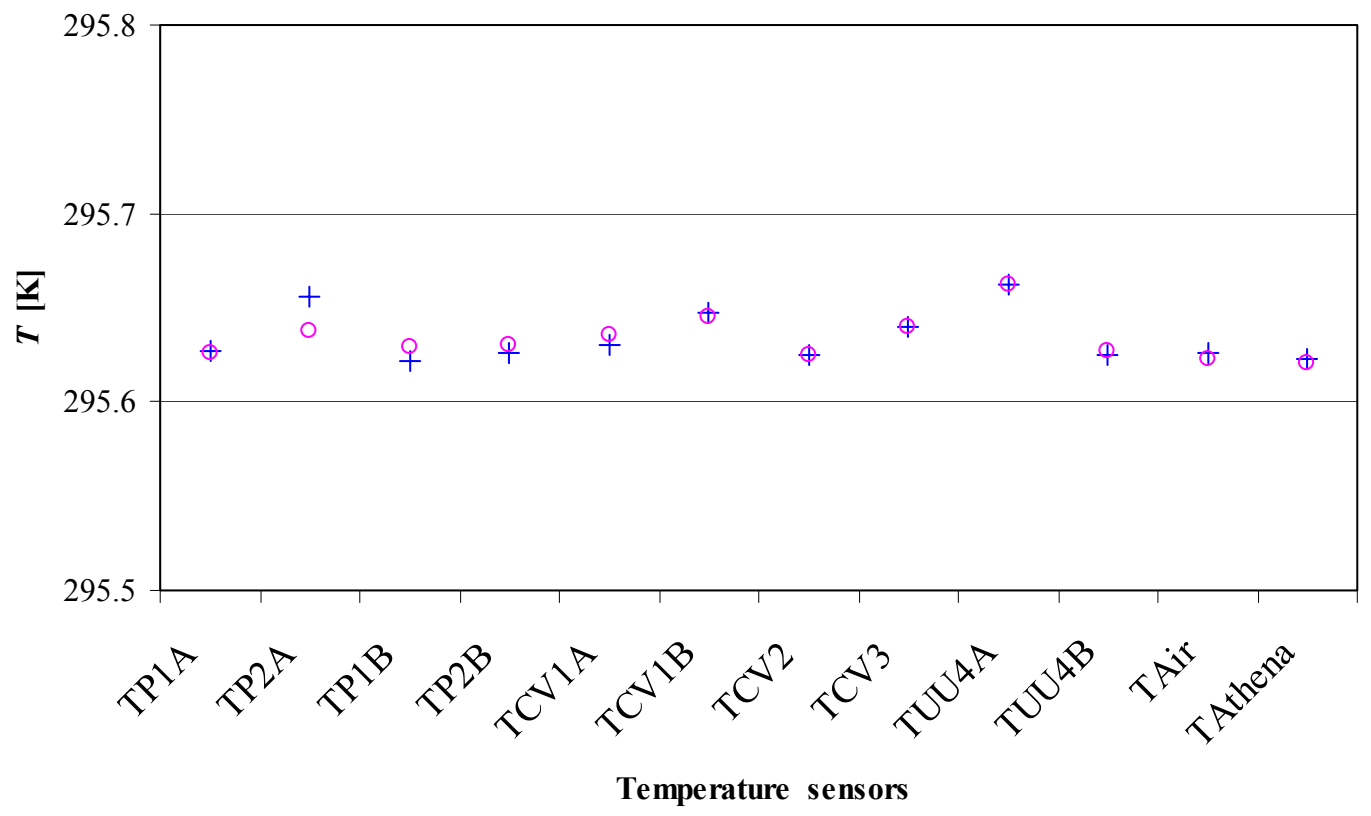

Figure 6. Temperature measurements made by the 12 sensors in the HLFS on two different occasions. Temperature uniformity along the flow path is less than $0.1 \mathrm{~K}$.

\section{The Connecting Volume}

The connecting volume is modeled using the following equation:

$$
V_{C V}=\pi d_{C V}^{2} l_{C V} / 4
$$

In (25), $d_{C V}$ is the averaged internal diameter of the connecting pipe and $l_{C V}$ is its length. There is significant uncertainty associated with the estimation of the quantities needed to precisely compute the connecting volume: piping inside diameters, piping lengths, internal volumes of the valves and elbows, the dead volume in the prover, the extra connecting volumes associated with the piping used for different MUTs, etc. However, as shown in Table 2 below, the sensitivity of the connecting volume determination on the computed result for the volumetric flow through the MUT is quite small. The large uncertainty of $11.6 \%$ in the connecting volume will result only $5.8 \times 10^{-5} \%$ of flow uncertainty. This is because the change in density of the fluid in the connecting volume during a flow measurement is small (the temperature profile is quite stable) and because the connecting volume is small in size compared to the volume swept out by the piston. Thus, the flow measurement uncertainty is relatively insensitive to the rather large uncertainty in the connecting volume size. Furthermore, the uncertainties of the dimension changes due to thermal expansion are even smaller and are neglected. 


\section{Time of Piston Displacement}

The uncertainty of the measurements of time can be separated into two parts: a) that due to the reference clock (including calibration errors and temperature effects) and b) that due to quantization errors. The time base oscillators are periodically calibrated by two reference counters that are traceable to NIST time and frequency standards. Table B7, Appendix B shows sample calibration results for the two oscillators in the HLFS. The Type A uncertainty of the HLFS oscillators used in this uncertainty analysis is $0.31 \mu \mathrm{Hz} / \mathrm{Hz}$ and the Type B uncertainty is $1.55 \mu \mathrm{Hz} / \mathrm{Hz}$. These oscillator calibration uncertainties are larger than the quantization uncertainties discussed below.

Figure 7 illustrates that the quantization error for a generic timed interval will be smaller than or equal to one time reference unit $( \pm \Delta t)$. The true time, $t_{\text {true }}$, is marked by the start and stop times, $t_{1}$ and $t_{3}$. The data acquisition system obtains the measured time, $t_{\text {meas }}$, by counting the number of rising edges, $n$, from the reference clock (nominally $1 \mathrm{MHz}$ in this case) between $t_{2}$ and $t_{4}$ and multiplying $n$ by the reference time unit $(1 \mu \mathrm{s})$. The timing errors at the start and end of the measurement $\left(\delta_{s}\right.$ and $\delta_{e}$ ) can each be between zero and one time unit in magnitude. The resulting difference between the true and measured times is \pm one time reference unit. The start and end timing errors each have rectangular probability distributions and the difference between them (the quantization error) has a standard uncertainty of $\Delta t / \sqrt{6}$. Although the example uses rising edges for triggering, this analysis is equally valid for falling edges. It does assume that no pulse is missed by the counter.

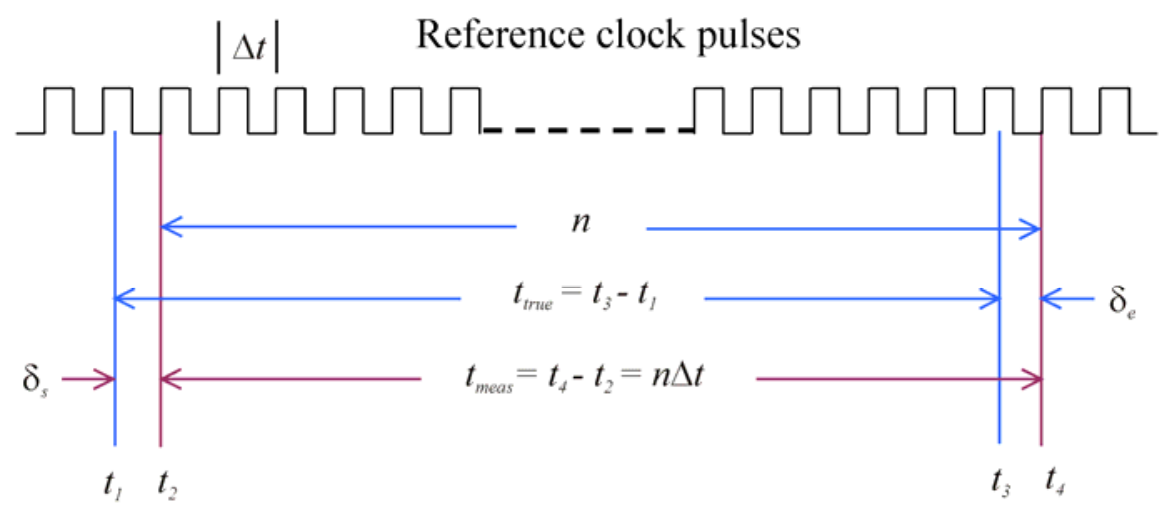

Figure 7. A sketch showing how reference clock pulses are used to time a generic interval and how the timing procedure leads to an quantization error of \pm one time reference unit $( \pm \Delta t)$.

Figure 8 illustrates the application of the reference clock to measurement of the time for the piston to travel a selected distance, as indicated by a selected number of pulses output by one of the encoders. The figure also illustrates the measurement of the frequency output by a MUT. Once the test conditions have reached steady state (at time $t_{0}$ ), the next rising edge output by the encoder is used to commence the counting of rising edges from the reference clock. After the predetermined number $\left(n_{E}\right)$ of encoder pulses has been registered by a counter, the counting of reference clock pulses is stopped, and the total $(n)$ is multiplied by $\Delta t$ to obtain the time required 
by the piston to travel the prescribed distance $\left(t_{\text {Emeas }}\right)$. As for the generic case, this time has a standard uncertainty due to quantization of $\Delta t / \sqrt{6}$.

The frequency of the flowmeter output, $f_{T}$, is calculated by dividing the total number of pulses output $\left(n_{T}\right)$ by the time between two rising edges of the flowmeter output $\left(t_{\text {Tmeas }}\right)$ that occur immediately after the encoder rising edges that mark the start and stop of the piston travel time. Note that the encoder time is measured independently from the flowmeter time and while they are very nearly coincident, they are not necessarily equal in duration. As for the previous cases, the flowmeter pulse totalization time has quantization uncertainty of $\Delta t / \sqrt{6}$.

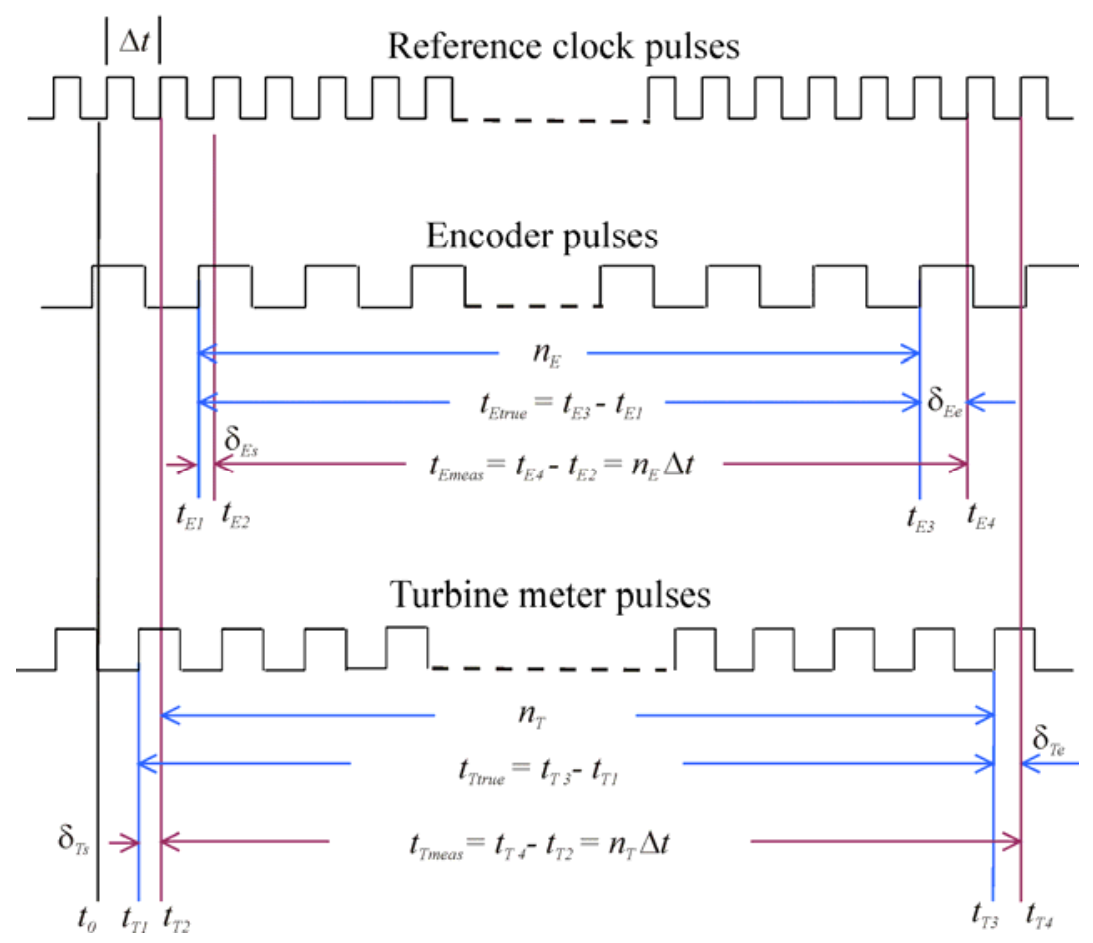

Figure 8. Diagram of the process for counting and timing the pulses from the encoder that measures piston displacement and from a pulse generating MUT (a turbine meter) and their quantization errors.

The HLFS has redundancy in the encoders and oscillators in order to avoid miscounting pulses and to allow internal validation of measurements. The prover system uses two encoders ( 1 and 2), each providing two pulse outputs (A and B). Output A originates from the leading edges of the encoder pulses and output $\mathrm{B}$ indicates the trailing edges. Therefore, a total of four chronometries $(1 \mathrm{~A}, 1 \mathrm{~B}, 2 \mathrm{~A}$, and $2 \mathrm{~B})$ are used to measure the piston travel and thereby improve the accuracy of the measured collection time. Additionally, the HLFS uses two oscillators to measure the piston travel interval. The first oscillator is used to measure time for chronometries $1 \mathrm{~A}$ and $2 \mathrm{~A}$ and the second oscillator operates on $1 \mathrm{~B}$ and $2 \mathrm{~B}$. The Type $\mathrm{B}$ uncertainties of the clocks are assumed to be fully correlated between clocks operated by the same oscillator. Increasing the number of chronometries or measurements does not improve the measurement uncertainty when the uncertainties are fully correlated. 


\section{Fluid Properties}

As indicated in (15), the thermal expansion of the fluid, and not the density itself, affects the volumetric flow determination. The physical property values involved in the expression for fluid density as a function of temperature are periodically determined off-line, using an oscillating tube densitometer (calibrated with distilled water and NIST Standard Reference Materials). A sample set of measured fluid density and the thermal expansion coefficient are shown in Table B9, Appendix B.

Likewise, the fluid kinematic viscosity does not directly affect the flow results in this type of calibrator. However, depending on the type of MUT, the fluid kinematic viscosity can affect the flowmeter output. It has been shown that one can obtain improved flow measurement performance when calibration results are expressed in non-dimensional parameters, such as Strouhal and Roshko numbers [4]. Using these, variations in the fluid temperature, kinematic viscosity, and/or density, from those used during the calibration conditions, can be compensated for. By anticipating the use of these non-dimensional parameters, our uncertainty analysis should apply to a wide range of fluid kinematic viscosity (i.e., 0.5 centistokes to 2 centistokes). For the report of calibration, the fluid viscosities are periodically measured using a capillary viscometer (Schott AVS 440), which measures the time required for an amount of fluid to flow through a capillary tube of known diameter and length. Table B9, Appendix B shows a typical set of viscosity data.

\subsection{Propagating Components of Uncertainty}

Referring to the graphic representation of the uncertainty analysis, given in Fig. 5 and the uncertainty propagation equation (19) given above, the uncertainty of the sub-measurements must be assessed before the flow uncertainty can be estimated. This process is propagated throughout all the measurement components until the uncertainties of the desired quantities are obtained. The details of the calculations of the propagation calculations for the submeasurements are given in Appendix B. Table B2 is for the temperature measurement uncertainties, Table B6 is for diameter, piston displacement, and volume measurements, and Table B8 is for the encoder timing uncertainty.

After the uncertainties of the sub-measurements are obtained, the one-standard-deviation, $(k=1)$, uncertainty for the volumetric flow of $3.0 \mathrm{~L} / \mathrm{min}(0.8 \mathrm{gal} / \mathrm{min})$, as given in (15), is obtained and shown in Table 2. The second column in Table 2 lists the nominal mean values of the measurement components. The third and fourth columns list their Type A and Type B uncertainties, respectively. The fifth and sixth columns list the same uncertainties as a percent of the mean. The seventh column lists the non-dimensional sensitivity coefficients of the component to the flow. The eighth and ninth columns list the flow uncertainty, in percent, due to the sub-component. These data show that the primary contribution to the flow uncertainty is the uncertainty of the piston displaced volume, $\Delta V_{P}(0.0021 \%)$ and to the temperature difference $\Delta T_{P, M U T}(-0.0031 \%)$ while the uncertainty contribution due to the connecting volume uncertainty is very small $(5.8 \mathrm{E}-5 \%)$. The total uncertainties of the flow are $0.0007 \%$ and $0.0037 \%$ for the Type A and Type B, respectively. 
Table 2. Volumetric flow rate uncertainty propagation using (15).

\begin{tabular}{ccccccccc}
\hline & & & & $u_{A}$ & $u_{B}$ & & $Q_{i} u_{A}$ & $Q_{i} u_{B}$ \\
& mean & $u_{A}$ & $u_{B}$ & {$[\%]$} & {$[\%]$} & $c_{i Q}$ & {$[\%]$} & {$[\%]$} \\
\hline$\Delta V_{P}\left[\mathrm{~cm}^{3}\right]$ & 1514.9 & 0.0006 & 0.0317 & $3.9 \mathrm{E}-05$ & 0.0021 & 1.00 & $3.9 \mathrm{E}-05$ & 0.0021 \\
$t[\mathrm{~s}]$ & 30.0 & $5.2 \mathrm{E}-06$ & $3.3 \mathrm{E}-05$ & $1.7 \mathrm{E}-05$ & $1.1 \mathrm{E}-04$ & -1.00 & $-1.7 \mathrm{E}-05$ & $-1.1 \mathrm{E}-04$ \\
$\alpha[1 / \mathrm{K}]$ & 0.00098 & 0 & $2.7 \mathrm{E}-06$ & 0 & 0.2768 & $-3.4 \mathrm{E}-05$ & 0 & $-9.5 \mathrm{E}-06$ \\
$\alpha_{S}[1 / \mathrm{K}]$ & $1.7 \mathrm{E}-05$ & 0 & $3.4 \mathrm{E}-07$ & 0 & 2.00 & $-1.3 \mathrm{E}-07$ & 0 & $-2.6 \mathrm{E}-07$ \\
$V_{C V}\left[\mathrm{~cm}^{3}\right]$ & 195.5 & 0 & 22.73 & 0 & 11.6 & $4.9 \mathrm{E}-06$ & 0 & $5.8 \mathrm{E}-05$ \\
$\Delta T_{P, M U T}[\mathrm{~K}]$ & 0.040 & 0.001 & 0.031 & 3.24 & 77.5 & $-3.9 \mathrm{E}-05$ & $-1.3 \mathrm{E}-04$ & $-3.1 \mathrm{E}-03$ \\
$\Delta T_{C V}[\mathrm{~K}]$ & 0.040 & 0.057 & 0 & 143 & 0 & $5.1 \mathrm{E}-06$ & $7.3 \mathrm{E}-04$ & 0 \\
$\Delta T_{C P}[\mathrm{~K}]$ & 0.020 & 0.049 & 0 & 243 & 0 & $-1.3 \mathrm{E}-07$ & $-3.2 \mathrm{E}-05$ & 0 \\
$Q_{M U T}\left[\mathrm{~cm}^{3} / \mathrm{s}\right]$ & 50.49 & 0.0004 & 0.0019 & & & & $\mathbf{0 . 0 0 0 7}$ & $\mathbf{0 . 0 0 3 7}$ \\
\hline
\end{tabular}

Using the same method, the uncertainties for other flows were calculated. Table 3 shows the results for a range of flows and the worst-case scenario for the total uncertainty for these flows. The worst-case scenario values are then used for the uncertainty specification for the 2 L HLFS:

$$
u_{A}=0.0018 \% \text { and } u_{B}=0.0037 \%
$$

Table 3. Uncertainties of the HLFS for several flows.

\begin{tabular}{cccccc}
\hline$Q$ & $t_{c}$ & $\Delta V_{P}$ & $\Delta L$ & $u_{A}$ & $u_{B}$ \\
\hline$[\mathrm{L} / \mathrm{min}]$ & {$[\mathrm{s}]$} & {$\left[\mathrm{cm}^{3}\right]$} & {$[\mathrm{cm}]$} & {$[\%]$} & {$[\%]$} \\
\hline 0.2 & 180 & 608 & 15 & 0.0018 & 0.0037 \\
0.4 & 180 & 1215 & 30 & 0.0009 & 0.0037 \\
0.8 & 113 & 1515 & 37.4 & 0.0007 & 0.0037 \\
1.5 & 60 & 1515 & 37.4 & 0.0007 & 0.0037 \\
3.0 & 32 & 1515 & 37.4 & 0.0007 & 0.0037 \\
5.0 & 18 & 1515 & 37.4 & 0.0007 & 0.0037 \\
\hline \multicolumn{7}{c}{ Max } & & 0.0018 & 0.0037 \\
\hline
\end{tabular}

\subsection{Combined and Expanded Uncertainty}


In accordance with [8], the combined standard uncertainty for the measurement system, is given by $u_{C}=\sqrt{u_{A}^{2}+u_{B}^{2}}$. That is:

$$
u_{C}=\sqrt{(0.0018)^{2}+(0.0037)^{2}} \%=0.0041 \%
$$

The approximate confidence level of the result given above is $68 \%$. When a coverage factor of $k=2$ is used to convert the combined standard uncertainty to an expanded uncertainty, with an approximate $95 \%$ level of confidence, the expanded uncertainty becomes:

$$
U= \pm k u_{C}=0.0082 \%<0.01 \%
$$

\subsection{Uncertainty of Meter Under Test}

The uncertainty analysis reported above is for the respective piston-cylinder and associated connecting volume for the prover of the HLFS. The uncertainty analysis for the MUT results will depend on the flowmeter type and the associated instruments used. As indicated above, improved flow measurement performance can be obtained by using non-dimensional parameters.

In addition to the system uncertainty discussed above, the uncertainty for the MUT should also include the standard deviation for replicated flow calibration results (i.e., reproducibility") [1]. Reproducibility data is used because flowmeter users need to know the short term stability (i.e., repeatability ${ }^{\vartheta}$ and hysteresis of their instruments. The long term stability of the MUT is also important as are reproducibility when turned-off and turned-on, and the day to day changes in its performance. These types of reproducibility errors can be an order of magnitude larger than the MUT's repeatability errors. The replicated uncertainty of the MUT is ascertained from multiple calibration results. By using this method, the total uncertainty of the calibration data for the test meter will include both contributions from the calibration system and MUT.

\footnotetext{
* Reproducibility is defined as the closeness of the agreement between the results of measurements of the same measurand carried out under changed conditions of measurement [1].

${ }^{\diamond}$ Repeatability is defined as the closeness of the agreement between the results of successive measurements of the same measurand carried out under the same conditions of measurement [1].

Hysteresis is defined as the closeness of the agreement between the results of measurements of the same measurand whether the value of the measurand is approached from a higher or lower values along its range (it is a form of reproducibility [1])
} 


\subsection{References}

[1] ISO, "International Vocabulary of Basic and General Terms in Metrology," 2nd ed., (International Organization for Standardization, Geneva, Switzerland, 1993).

[2] G. E. Mattingly, "Flow Measurement Proficiency Testing," ISA Flow Measurement, 2nd ed., Practical Guides for Measurement and Control, Ch. 28 (Instrument Society of America, NC, 2001).

[3] T. T. Yeh, P.I. Espina, G.E. Mattingly, and N.R. Briggs, "An Uncertainty Analysis of a NIST Hydrocarbon Liquid Flow Calibration Facility," Proceedings of HT/FED'04, HTFED2004-56790, 2004 Heat Transfer/Fluids Engineering Summer Conference, Chariotte, North Carolina, USA, July 11-15, 2004.

[4] G. E. Mattingly, "The Characterization of a Piston Displacement-Type Flowmeter Calibration Facility and the Calibration and Use of Pulsed Output Type Flowmeters," NIST J. of Res., 975 pp. 509-531, 1992.

[5] J. D. Wright and G. E. Mattingly, "NIST Calibration Services for Gas Flow Meters: Piston Prover and Bell Prover Gas Flow Facilities", NIST SP 250-49 (National Institute of Standards and Technology, Gaithersburg, MD, 1998).

[6] API, "Manual of Petroleum Measurement Standards, Chapter 4.3: Proving Systems - Small Volume Provers", 1st ed. (American Petroleum Institute, Washington, D.C., 1993).

[7] B. N. Taylor and C. E. Kuyatt, "Guidelines for the Evaluating and Expressing the Uncertainty of NIST Measurement Results", NIST TN-1297 (National Institute of Standards and Technology, Gaithersburg, MD, 1994).

[8] ISO, "Guide to the Expression of Uncertainty in Measurement" (International Organization for Standardization, Geneva, Switzerland, 1993).

[9] Coleman, H. W. and Steele, W. G., "Experimentation and Uncertainty Analysis for Engineers," 2nd ed., (John Wiley and Sons, 1999). 


\section{Appendix A: Volume Balance Method}

An alternate method for correcting fluid volume in the connecting volume is the volume balance method. For a given fluid, the change of its volume, $\Delta V_{F}$, is given by the final fluid volume, $V_{F f}$, minus the initial fluid volume, $V_{F i}$, or,

$$
\Delta V_{F}=V_{F f}-V_{F i}
$$

Using Figure A1 as a reference, the initial fluid volume can be expressed as,

$$
V_{F i}=\left(V_{P}+V_{C V}+V_{M U T}\right)_{i}
$$

while the final fluid volume is given by,

$$
V_{F f}=\left(V_{P}+V_{C V}+V_{M U T}\right)_{f}+V_{L}
$$

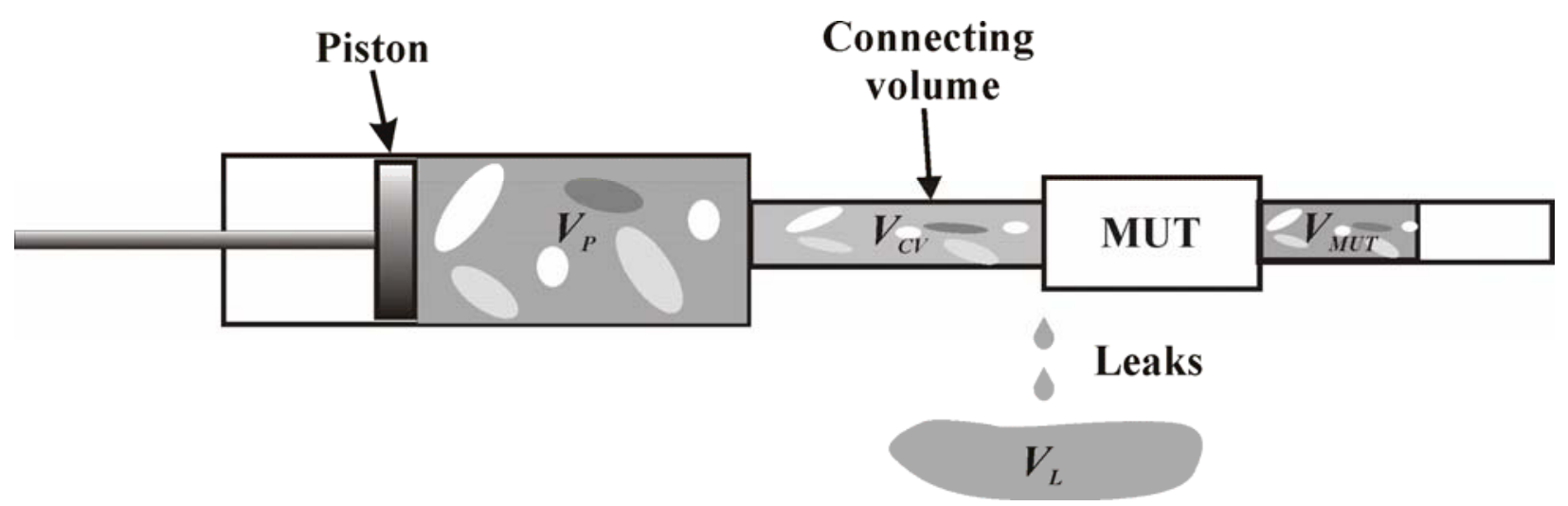

Figure A1. Balance of fluid quantities throughout the control volume. Different colors denote different fluid temperatures or different fluid densities.

In (A2) and (A3), $V_{P i}$ and $V_{P f}$ are the fluid volumes contained in the piston prover at the initial (start) and final (stop) times, respectively. Similarly, $V_{C V i}$ and $V_{C V f}$ are the fluid volumes contained within the connecting pipe at the initial and final times, respectively. $V_{M U T i}$ and $V_{\text {MUTf }}$ are the fluid volumes discharged through the MUT at the initial and final times, respectively, and $V_{L}$ is the volume of any leaked fluid. As discussed above, the leakage $V_{L}$ will be assumed to be zero and not considered further in this discussion.

Combining (A1-A3), we obtain an expression for the net fluid volume discharged through the meter,

$$
\Delta V_{M U T}=V_{M U T f}-V_{M U T i}=\Delta V_{F}+\Delta V_{P}-\Delta V_{C V}
$$


where, $\Delta V_{P}=V_{P i}-V_{P f}$ is the net fluid volume displaced by the piston, and $\Delta V_{C V}=V_{C V f}-V_{C V i}$ is the change of the connecting volume due to thermal expansion of the connecting pipe. Next, we shall discuss each of the volume change terms.

The volume change of the connecting volume due to the temperature change, $\Delta T_{C P}$ can be expressed as,

$$
\Delta V_{C V}=V_{C V} 3 \alpha_{S} \Delta T_{C P}
$$

where $V_{C V}$ is the average connecting volume.

In contrast to $\Delta V_{C V}$, the volume change of the fluid, $\Delta V_{F}$, is more difficult to estimate. As seen before, because the modulus of elasticity of the fluid is very large ( $2 \mathrm{GPa}$ ) and the working pressure is small in comparison $(80 \mathrm{kPa})$, and fairly constant $(\Delta P<80 \mathrm{~Pa})$, the density of the liquid can be assumed to be function of fluid temperature only. Thus, for a given mass of fluid, changes in fluid volume will only be due to changes in fluid temperature. For a small change of temperature $\Delta T$, it can be shown that:

$$
\rho=\rho_{0}(1-\alpha \Delta T), \Delta \rho=-\rho_{0} \alpha \Delta T, \text { and } V=V_{0} \alpha \Delta T
$$

If the fluid volume is divided into small volume elements, $V_{j}$ (see Figure 5), such that each small volume has constant fluid temperature, $T_{j}$, the volume change of each element can be expressed as,

$$
\Delta V_{j}=V_{j} \alpha \Delta T_{j}=\alpha \Delta H_{j} /\left(\rho c_{P}\right)
$$

where $\Delta H_{j}$ is the heat transferred to the $j$-th element of the fluid, and $c_{P}$ is the specific heat capacity of the fluid. By summing over all elements, the total (or net) volume increase of the fluid is,

$$
\Delta V_{F}=\sum \Delta V_{j}=\left(\alpha / \rho c_{P}\right) \sum \Delta H_{j}=\left(\alpha / \rho c_{P}\right) \Delta H
$$

where $\Delta H$ is the total (or net) heat addition to the fluid in the connecting volume during the calibration time $t_{c}$.

From (A8), it is worth noting that heat transfer between cold and hot pockets of fluid does not change the total volume of the fluid. Only net heat addition to the fluid will change the total fluid volume. Thus, if there is no net heat addition to the fluid, there will be no change in the fluid volume.

In our calibrator, some heat addition is inevitable due to viscous dissipation as the fluid moves through the pipe. There is also some heat transfer between the pipe and fluid. Thus, the net heat addition is normally a function of flow and the temperature difference between the calibrator and the ambient air. Insulating the connecting pipe can reduce the heat transfer to or from between the room, thus normally reducing the amount of heat transfer between the pipe and fluid. Also, operating the calibrator near room temperature will reduce this heat transfer. Equation (A8) further shows that, large temperature coefficients, $\alpha$, will lead to large volume increases, while large fluid densities, $\rho$, or specific heat capacities, $c_{P}$, will result in smaller volume increases. 
According to the conservation of energy, the heat addition, $\Delta H$ for the liquid is related to the average temperature rise of the fluid as it moves through the connecting volume, $\Delta T_{H, C V}$, as:

$$
\Delta H=\rho c_{P} \Delta V_{P} \Delta T_{H, C V}
$$

Under normal operational conditions, the heat addition is a system characteristic which should be fairly constant.

By combining (A8) and (A9) and eliminating the heat addition, $\Delta H$, the fluid volume change, $\Delta V_{F}$, becomes:

$$
\Delta V_{F}=\Delta V_{P} \alpha \Delta T_{H, C V}
$$

This equation shows that the fluid volume increase depends only on the temperature rise, $\Delta T_{H, C V}$, and the thermal expansion coefficient of the fluid, $\alpha$, but not on the fluid specific heat capacity explicitly.

By combining (A4), (A5) and (A10) and dividing by the calibration time, $t_{c}$, we obtain the average fluid volume flowing through the MUT. That is,

$$
Q_{M U T}=\frac{\Delta V_{M U T}}{t_{c}}=\frac{1}{t_{c}}\left[\Delta V_{P}\left(1+\alpha \Delta T_{H, C V}\right)-3 V_{C V} \alpha_{S} \Delta T_{C P}\right]
$$

Once again, it is worth noting that the fluid density does not directly affect the volumetric flow calculation. Next, we will discuss how we determine the fluid temperature rise in the connecting volume during the calibration.

The fluid passing through the MUT is not exactly the same fluid that is being discharged from the prover. For the sake of discussion, the fluid that affects the average temperature rise can be divided into three parts: I, II and III. Figure A2 shows the sketch of the distribution of the three parts of fluid and the temperature variations as functions of time at MUT, $T_{M U T}$, and at piston, $T_{P}$. Part I fluid is the fluid found in the connecting volume at the time the calibration starts. During the calibration period, this fluid is pushed through the MUT. All of the fluid in Part II is displaced by the piston and passes through the MUT during the calibration period. The fluid in Part III is also displaced by the piston but does not reached the MUT before the calibration ends (i.e., still in the connecting volume). The determination of the temperature rise for the fluid in part II is simple, while those for part I and III are more complex. The average temperature rise, $\Delta T_{H, C V}$ is thus the weighted average temperature rise of the three parts of fluid.

For each fluid element, its temperature rise is the difference of its temperature at the initial and final times. To help illustrate this point, the fluid temperature at position $x$ and time $t$ is given as $T(x, t)$. Here, $x$ is the distance measured from the piston, i.e., $x=0$ at the piston and $x=x_{M U T}$ at the MUT. The temperature at two special locations, the MUT and the piston, (shown on Figure A2) are:

$$
T_{M U T}(t)=T\left(x_{M U T}, t\right) \text { and } T_{P}(t)=T(0, t)
$$

and the temperatures at two special times, the initial and final times, are:

$$
T_{i}(x)=T(x, 0) \text { and } T_{f}(x)=T\left(x, t_{c}\right)
$$

The position of a fluid element at time $t, x$, can be expressed by 


$$
x=x_{0}+U_{C V} t
$$

where $x_{0}$ is the initial position of the fluid, and $U_{C V}=4 Q_{M U T} / \pi d_{C V}^{2}$ is the average fluid velocity in the connecting volume. Here, $d_{C V}$ is the inner diameter of the connecting pipe.
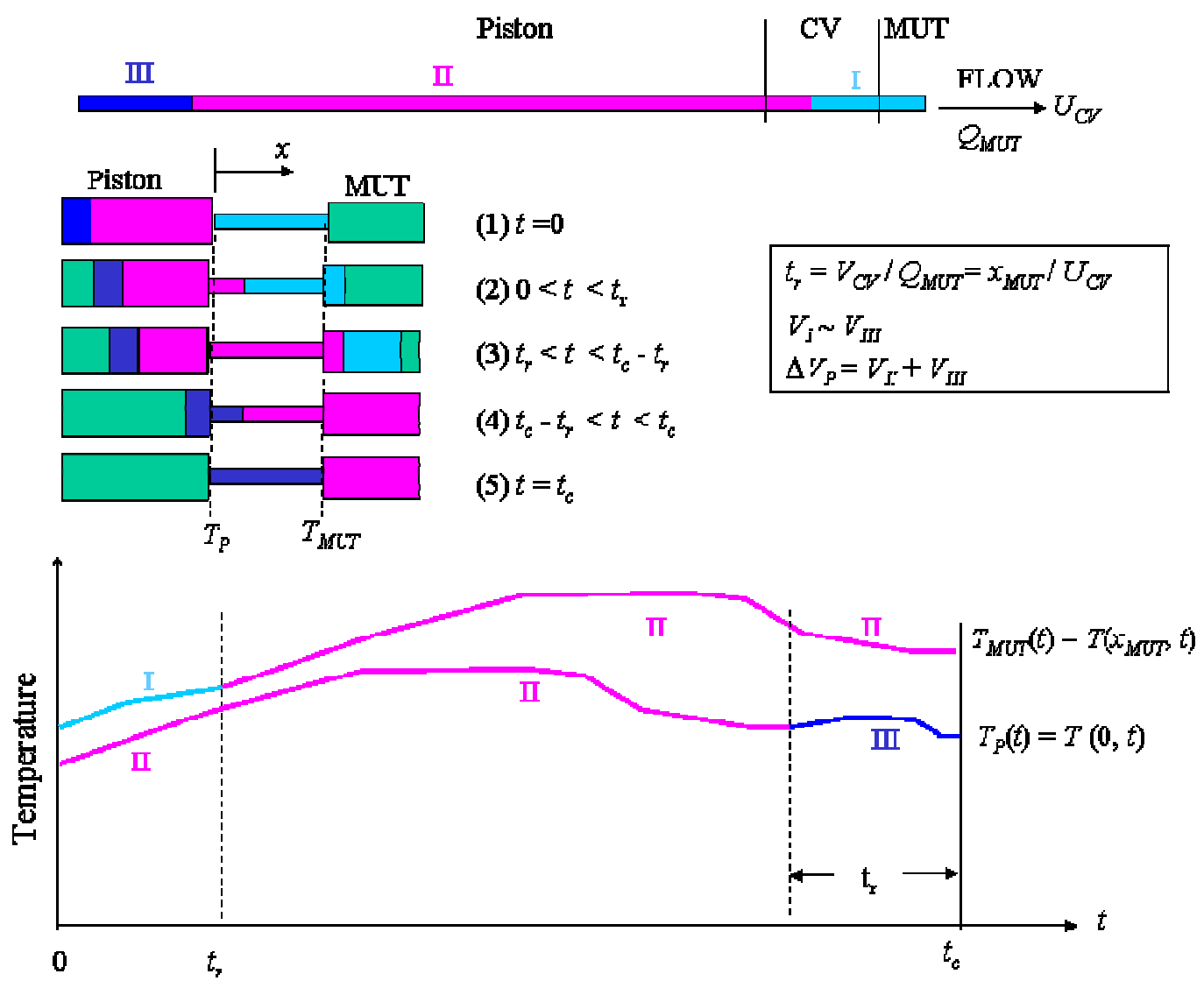

Figure A2. Sketch and estimation of the average temperature rise of fluid as it moves through the connecting volume. $U_{C V}$ is the average flow velocity in the connecting volume. $T_{M U T}(t)$ and $T_{P}(t)$ are the temperature as functions of time at the MUT and at the prover, respectively.

As referred to Figure A2, we have the average temperature rise

$$
\Delta T_{H, C V}=\frac{1}{t_{c}}\left(\int_{0}^{t_{r}} \Delta T_{I} d t+\int_{t_{r}}^{t_{c}} \Delta T_{I I} d t+\int_{t_{c}-t_{r}}^{t_{c}} \Delta T_{I I I} d t\right)
$$

where

$$
\Delta T_{I}=T_{M U T}(t)-T\left(x_{M U T}-U_{C V} t, 0\right)
$$

is the temperature rise of the fluid element $I$ located at the MUT and time $t$; 


$$
\Delta T_{I I}=T_{M U T}(t)-T_{P}\left(t-t_{r}\right)
$$

is the temperature rise of the fluid element $I I$; and

$$
\Delta T_{I I I}=T\left(U_{C V}\left(t_{c}-t\right), t_{c}\right)-T_{P}(t)
$$

is the temperature rise of the fluid element $I I I$ located at the Piston and time $t$. Inserting (A16 A18) into (A15), we have

$$
\begin{aligned}
& \Delta T_{H, C V}= \frac{1}{t_{c}}\left(\begin{array}{c}
\int_{0}^{t_{r}}\left[T_{M U T}(t)-T\left(x_{M U T}-U_{C V} t, 0\right)\right] d t+\int_{t_{r}}^{t_{c}}\left[T_{M U T}(t)-T_{P}\left(t-t_{r}\right.\right. \\
\quad+\int_{t_{c}-t_{r}}^{t_{c}}\left[T\left(U_{C V}\left(t_{c}-t\right), t_{c}\right)-T_{P}(t)\right] d t
\end{array}\right. \\
&=\frac{1}{t_{c}}\left(\begin{array}{c}
\int_{0}^{t_{c}} T_{M U T}(t) d t-\int_{0}^{t_{c}} T_{P}(t) d t \\
\left.\quad-\int_{0}^{t_{r}} T\left(x_{M U T}-U_{C V} t, 0\right) d t+\int_{t_{c}-t_{r}}^{t_{c}} T\left(U_{C V}\left(t_{c}-t\right), t_{c}\right) d t\right)
\end{array}\right) \\
&=T_{M U T, m}-T_{P, m}+\frac{1}{t_{c}}\left[\int_{0}^{x_{M U T}} T\left(x, t_{c}\right) \frac{d x}{U_{C V}}-\int_{0}^{x_{M U T}} T(x, 0) \frac{d x}{U_{C V}}\right] \\
&=-\Delta T_{P, M U T}+\frac{t_{r}}{t_{c}}\left(T_{C V, f}-T_{C V, i}\right) \\
&=-\Delta T_{P, M U T}+\frac{V_{C P}}{\Delta V_{P}} \Delta T_{C V}
\end{aligned}
$$

Thus, when substituting (A19) into (A11), we obtain the average fluid volume flowing through the MUT as,

$$
Q_{M U T}=\frac{1}{t_{c}}\left[\Delta V_{P}\left(1-\alpha \Delta T_{P, M U T}\right)+V_{C V}\left(\alpha \Delta T_{C V}-3 \alpha_{S} \Delta T_{C P}\right)\right]
$$

This equation is exactly (15), which was obtained from the mass conservation method. 


\section{Appendix B: Supporting Data for Uncertainty Analysis}

B1. Temperature measurement uncertainty components.

Table B1. Thermistor calibration uncertainties: the standard deviation of repeated readings $\left(u_{A}\right)$ and the difference from the reference sensor $\left(u_{B}\right)$. The maximum values of all sensors $\left(T_{U U 4 A}\right)$ were used for the uncertainties of the thermistors; $u_{A}=0.041 \mathrm{~K}$ and $u_{B}=0.031 \mathrm{~K}$.

\begin{tabular}{|c|c|c|}
\hline & \multicolumn{2}{|c|}{$T-T_{\text {ref }}$} \\
& $u_{A}$ & $u_{B}$ \\
& {$[\mathrm{~K}]$} & {$[\mathrm{K}]$} \\
$T_{P 1 A}$ & 0.007 & -0.015 \\
$T_{P 2 A}$ & 0.026 & 0.010 \\
$T_{U U 4 A}$ & 0.041 & 0.031 \\
$T_{C V 1 B}$ & 0.031 & 0.006 \\
$T_{C V 1 A}$ & 0.017 & -0.005 \\
$T_{U U 4 B}$ & 0.032 & -0.012 \\
$T_{P 1 B}$ & 0.010 & -0.012 \\
$T_{P 2 B}$ & 0.009 & -0.011 \\
$T_{C V 2}$ & 0.011 & -0.014 \\
$T_{C V 3}$ & 0.004 & 0.000 \\
$T_{\text {Air }}$ & 0.029 & -0.019 \\
$T_{\text {Athena }}$ & 0.027 & -0.019 \\
Max & 0.041 & 0.031 \\
\hline
\end{tabular}

Table B2. Temperature measurement uncertainties.

\begin{tabular}{|c|c|c|c|c|}
\hline \multicolumn{5}{|c|}{$T_{P x, m} n>=1000, x=A$ or $B$} \\
\hline & mean & $\mathrm{n}$ & $u_{A}$ & $u_{B}$ \\
\hline$T_{P x}[\mathrm{~K}]$ & 295.2 & 1000 & 0.041 & 0.031 \\
\hline$T_{P x, m}[\mathrm{~K}]$ & 295.2 & & 0.001 & 0.031 \\
\hline \multicolumn{5}{|c|}{$T_{M U T x, m} n>=1000, x=A$ or $B$} \\
\hline & mean & $\mathrm{n}$ & $u_{A}$ & $u_{B}$ \\
\hline$T_{M U T x}[\mathrm{~K}]$ & 295.2 & 1000 & 0.041 & 0.031 \\
\hline$T_{M U T x, m}[\mathrm{~K}]$ & 295.2 & & 0.001 & 0.031 \\
\hline \multicolumn{5}{|c|}{$T_{\text {Air, } m} n>=1000$} \\
\hline & mean & $\mathrm{n}$ & $u_{A}$ & $u_{B}$ \\
\hline$T_{\text {Air }}[\mathrm{K}]$ & 295.2 & 1000 & 0.041 & 0.031 \\
\hline
\end{tabular}



$T_{A i r, m}[\mathrm{~K}]$
295.2
0.001
0.031

$T_{S A}$ Temperature sensors used for spatial average

\begin{tabular}{clccc} 
& mean & $c_{i}$ & $u_{A}$ & $u_{B}$ \\
$T_{i}[\mathrm{~K}]$ & 295.2 & 1 & 0.041 & 0.031 \\
$E_{M a x}[\mathrm{~K}]$ & 0 & 1 & 0.1 & 0 \\
$T_{S A}[\mathrm{~K}]$ & 295.2 & \multicolumn{4}{c}{0.108} & 0.031 \\
\hline \multicolumn{5}{c}{$T_{P, x}=\left(T_{P A}+T_{P B}\right) / 2, x=i$ or $f$} \\
$T_{P A, x}[\mathrm{~K}]$ & mean & $c_{i}$ & $u_{A}$ & $u_{B}$ \\
$T_{P B, x}[\mathrm{~K}]$ & 295.2 & 0.5 & 0.108 & 0.031 \\
$T_{P, x}[\mathrm{~K}]$ & 295.2 & 0.5 & 0.108 & 0.031 \\
& 295.2 & & 0.076 & 0.022
\end{tabular}

\begin{tabular}{|ccccc|}
\hline \multicolumn{5}{c|}{$T_{P, m}=\left(T_{P A, m}+T_{P B, m}\right) / 2$} \\
$T_{P A, m}[\mathrm{~K}]$ & mean & $c_{i}$ & $u_{A}$ & $u_{B}$ \\
$T_{P B, m}[\mathrm{~K}]$ & 295.2 & 0.5 & 0.001 & 0.031 \\
$T_{P, m}[\mathrm{~K}]$ & 295.2 & 0.5 & 0.001 & 0.031 \\
& 295.2 & & 0.001 & 0.022
\end{tabular}

\begin{tabular}{|ccccc|}
\hline \multicolumn{5}{c|}{$T_{M U T, x}=\left(T_{\text {MUTA }}+T_{\text {MUTB }}\right) / 2, x=i$ or $f$} \\
& mean & $c_{i}$ & $u_{A}$ & $u_{B}$ \\
$T_{M U T A, x}[\mathrm{~K}]$ & 295.2 & 0.5 & 0.108 & 0.031 \\
$T_{M U T B, x}[\mathrm{~K}]$ & 295.2 & 0.5 & 0.108 & 0.031 \\
$T_{M U T, x}[\mathrm{~K}]$ & 295.2 & & 0.076 & 0.022
\end{tabular}

\begin{tabular}{|ccccc|}
\hline \multicolumn{5}{c|}{$T_{\text {MUT,m }}=\left(T_{M U T A, m}+T_{M U T B, m}\right) / 2$} \\
\\
mean & $c_{i}$ & $u_{A}$ & $u_{B}$ \\
$T_{M U T A, m}[\mathrm{~K}]$ & 295.2 & 0.5 & 0.001 & 0.031 \\
$T_{M U T B, m}[\mathrm{~K}]$ & 295.2 & 0.5 & 0.001 & 0.031 \\
$T_{M U T, m}[\mathrm{~K}]$ & 295.2 & & 0.001 & 0.022
\end{tabular}

\begin{tabular}{|ccccc|}
\hline \multicolumn{5}{c|}{$T_{C V I}=\left(T_{C V I A}+T_{C V 1 B}\right) / 2$} \\
$T_{C V I A}[\mathrm{~K}]$ & mean & $c_{i}$ & $u_{A}$ & $u_{B}$ \\
$T_{C V I B}[\mathrm{~K}]$ & 295.2 & 0.5 & 0.108 & 0.031 \\
& 295.2 & 0.5 & 0.108 & 0.031
\end{tabular}


$\begin{array}{llll}T_{C V 1}[\mathrm{~K}] & 295.2 & 0.076 & 0.022\end{array}$

\begin{tabular}{ccccc|}
\hline \multicolumn{4}{c}{$\Delta T_{P, M U T}=T_{P, m}-T_{M U T, m}$} \\
\hline & mean & $c_{i} i$ & $u_{A}$ & $u_{B}$ \\
$T_{P, m}[\mathrm{~K}]$ & 295.2 & 1 & 0.001 & 0.022 \\
$T_{M U T, m}[\mathrm{~K}]$ & 295.2 & -1 & 0.001 & 0.022 \\
$\Delta T_{P, M U T}[\mathrm{~K}]$ & 0.040 & & 0.001 & 0.031
\end{tabular}

\begin{tabular}{|ccccc|}
\hline \multicolumn{4}{c|}{$T_{C V, x}=\left(T_{P, x}+T_{C V I}+T_{C V 2}+T_{C V 3}+T_{M U T, x}\right) / 5, x=i$ or $f$} \\
\hline & mean & $c_{i}$ & $u_{A}$ & $u_{B}$ \\
$T_{P, x}[\mathrm{~K}]$ & 295.2 & 0.20 & 0.076 & 0.022 \\
$T_{C V 1}[\mathrm{~K}]$ & 295.2 & 0.20 & 0.076 & 0.022 \\
$T_{C V 2}[\mathrm{~K}]$ & 295.2 & 0.20 & 0.108 & 0.031 \\
$T_{C V 3}[\mathrm{~K}]$ & 295.2 & 0.20 & 0.108 & 0.031 \\
$T_{M U T, x}[\mathrm{~K}]$ & 295.2 & 0.20 & 0.076 & 0.022 \\
$T_{C V, x}[\mathrm{~K}]$ & 295.2 & & 0.040 & 0.012
\end{tabular}

\begin{tabular}{|clccc|}
\hline \multicolumn{5}{c|}{$\Delta T_{C V}=T_{C V, f}-T_{C V, i}$} \\
$T_{C V, f}[\mathrm{~K}]$ & mean & $c_{i}$ & $u_{A}$ & $u_{B}$ \\
$T_{C V, i}[\mathrm{~K}]$ & 295.2 & 1 & 0.040 & 0.012 \\
$\Delta T_{C V}[\mathrm{~K}]$ & 0.040 & & 0.057 & 0.012 \\
\end{tabular}

\begin{tabular}{|ccccc|}
\hline \multicolumn{5}{c|}{$T_{C P, x}=\left(T_{P, x}+T_{C V 1}+T_{C V 2}+T_{C V 3}+T_{M U T, x}+T_{A i r}\right) / 6, x=i$ or $f$} \\
$T_{P, x}[\mathrm{~K}]$ & mean & $c_{i}$ & $u_{A}$ & $u_{B}$ \\
$T_{C V 1}[\mathrm{~K}]$ & 295.2 & 0.1667 & 0.076 & 0.022 \\
$T_{C V 2}[\mathrm{~K}]$ & 295.2 & 0.1667 & 0.076 & 0.022 \\
$T_{C V 3}[\mathrm{~K}]$ & 295.2 & 0.1667 & 0.108 & 0.031 \\
$T_{M U T, x}[\mathrm{~K}]$ & 295.2 & 0.1667 & 0.076 & 0.022 \\
$T_{A i r}[\mathrm{~K}]$ & 295.2 & 0.1667 & 0.041 & 0.031 \\
$T_{C P, x}[\mathrm{~K}]$ & 295.2 & & 0.034 & 0.011
\end{tabular}




\begin{tabular}{|ccccc|}
\hline \multicolumn{5}{c|}{$\Delta T_{C P}=T_{C P, f}-T_{C P, i}$} \\
$T_{C P, f}[\mathrm{~K}]$ & mean & $c_{i}$ & $u_{A}$ & $u_{B}$ \\
$T_{C P, i}[\mathrm{~K}]$ & 295.2 & 1 & 0.034 & 0.011 \\
$\Delta T_{C P}[\mathrm{~K}]$ & 295.2 & -1 & 0.034 & 0.011 \\
\hline
\end{tabular}

\begin{tabular}{|ccccc|}
\hline \multicolumn{5}{c|}{$\Delta T_{D R}=T_{P, m}-T_{R D}$} \\
$T_{P, m}[\mathrm{~K}]$ & mean & $c_{i}$ & $u_{A}$ & $u_{B}$ \\
$T_{R D}[\mathrm{~K}]$ & 295.2 & 1 & 0.001 & 0.022 \\
$\Delta T_{D R}[\mathrm{~K}]$ & -0.08 & -1 & 0.010 & 0.010 \\
& & & 0.010 & 0.024
\end{tabular}

\begin{tabular}{|ccccc|}
\hline \multicolumn{5}{c|}{$\Delta T_{d R}=T_{P, m}-T_{R d}$} \\
$T_{P, m}[\mathrm{~K}]$ & mean & $c_{i}$ & $u_{A}$ & $u_{B}$ \\
$T_{R d}[\mathrm{~K}]$ & 295.2 & 1 & 0.001 & 0.022 \\
$\Delta T_{d R}[\mathrm{~K}]$ & 295.4 & -1 & 0.010 & 0.010 \\
& -0.21 & & 0.010 & 0.024
\end{tabular}

\begin{tabular}{|ccccc|}
\hline \multicolumn{5}{c|}{$\Delta T_{E R}=T_{A i r, m}-T_{R E}$} \\
$T_{A i r, m}[\mathrm{~K}]$ & mean & $c_{i}$ & $u_{A}$ & $u_{B}$ \\
$T_{R E}[\mathrm{~K}]$ & 295.2 & 1 & 0.001 & 0.031 \\
$\Delta T_{E R}[\mathrm{~K}]$ & 295.5 & -1 & 0.010 & 0.010 \\
& 1.000 & & 0.010 & 0.033
\end{tabular}


B2. Diameter, piston displacement, and volume measurement uncertainties.

Table B3. Shaft Diameter, $d_{R}$ and its uncertainty.

\begin{tabular}{|c|c|c|c|c|c|c|}
\hline Mitutoyo & Outside dia & ter micron & er Model: & ИDC-2"JT, & /n:293-37c & \\
\hline Standard & eference B & ks calibrat & by NIST & ecision En & neering D & \\
\hline & & Test 1 & Test 2 & Test 3 & Test 4 & Average \\
\hline & $T[\mathrm{C}]$ & 22.353 & 22.310 & 22.396 & 22.564 & 22.406 \\
\hline position \# & position & & & & & \\
\hline$[-]$ & {$[\mathrm{cm}]$} & {$[\mathrm{mm}]$} & {$[\mathrm{mm}]$} & {$[\mathrm{mm}]$} & {$[\mathrm{mm}]$} & \\
\hline 1 & 56.5 & 25.4446 & 25.4425 & 25.4437 & 25.4424 & \\
\hline 2 & 51.6 & 25.4428 & 25.4431 & 25.4432 & 25.4440 & \\
\hline 3 & 46.5 & 25.4431 & 25.4435 & 25.4439 & 25.4437 & \\
\hline 4 & 41.5 & 25.4402 & 25.4405 & 25.4406 & 25.4416 & \\
\hline 5 & 36.5 & 25.4413 & 25.4419 & 25.4418 & 25.4450 & \\
\hline 6 & 31.5 & 25.4400 & 25.4406 & 25.4413 & 25.4412 & \\
\hline 7 & 26.5 & 25.4412 & 25.4411 & 25.4403 & 25.4413 & \\
\hline 8 & 21.5 & 25.4399 & 25.4406 & 25.4414 & 25.4416 & \\
\hline 9 & 16.5 & 25.4402 & 25.4404 & 25.4405 & 25.4413 & \\
\hline 10 & 11.5 & 25.4399 & 25.4403 & 25.4404 & 25.4414 & \\
\hline 11 & 6.5 & 25.4384 & 25.4343 & 25.4391 & 25.4388 & \\
\hline & mean $d_{R}$ & 25.4411 & 25.4408 & 25.4415 & 25.4420 & 25.4413 \\
\hline & std unc & 5.38E-04 & 7.34E-04 & $4.76 \mathrm{E}-04$ & $5.04 \mathrm{E}-04$ & 0.0006 \\
\hline & & & & Ref gage & & $6.25 \mathrm{E}-05$ \\
\hline & & & & Combined & & 0.00057 \\
\hline
\end{tabular}


Table B4. Cylinder inside diameter, $D_{R}$ and its uncertainty.

\begin{tabular}{|c|c|c|c|c|c|}
\hline \multicolumn{6}{|c|}{ Mitutoyo 3-legged inner diameter micrometer, Model: IT-005D, s/n=10019027 } \\
Two Standard reference Rings calibrated by NIST Precision Engineering Division \\
\multicolumn{1}{|c|}{} & & Test 1 & Test 2 & Test 3 & Average \\
Position & $T[\mathrm{C}]$ & 22.199 & 22.189 & 22.464 & 22.284 \\
$\#$ & position & Gap & Gap & Gap & \\
{$[-]$} & {$[\mathrm{cm}]$} & {$[\mathrm{mm}]$} & {$[\mathrm{mm}]$} & {$[\mathrm{mm}]$} & \\
1 & 59.182 & -0.01711 & -0.01931 & -0.01632 & \\
2 & 54.182 & -0.01751 & -0.01992 & -0.01642 & \\
3 & 49.182 & -0.01912 & -0.01992 & -0.01722 & \\
4 & 44.182 & -0.01982 & -0.02092 & -0.01832 & \\
5 & 37.182 & -0.01501 & -0.01631 & -0.01502 & \\
6 & 30.182 & -0.01481 & -0.01681 & -0.01562 & \\
7 & 35.858 & -0.01462 & -0.01683 & -0.01842 & \\
8 & 28.858 & -0.01322 & -0.01643 & -0.01752 & \\
9 & 21.858 & -0.01882 & -0.02064 & -0.02192 & \\
10 & 16.858 & -0.01872 & -0.02024 & -0.02102 & \\
11 & 11.858 & -0.01702 & -0.01884 & -0.01932 & \\
12 & 6.858 & -0.01812 & -0.02024 & -0.02162 & \\
Mean gap & & -0.0170 & -0.0189 & -0.0182 & -0.0180 \\
std unc & & 0.00061 & 0.00051 & 0.00067 & 0.00060 \\
Ref ring & & 76.2056 & 76.2056 & 76.2056 & \\
Mean $D_{R}$ & & 76.1886 & 76.1867 & 76.1873 & 76.1875 \\
& & & Ref unc & & 0.00015 \\
& & & Combined unc & 0.00062 & \\
\hline
\end{tabular}


Table B5. Encoder constant, $K_{E R}$ and its uncertainty.

\begin{tabular}{|c|c|c|c|c|c|c|c|c|c|}
\hline $\begin{array}{l}\text { Standard } \\
\text { Ref unc = }\end{array}$ & $\begin{array}{l}\text { Laser In } \\
6.25 \mathrm{E}-\end{array}$ & $\begin{array}{l}\text { erferol } \\
\mathrm{mm}\end{array}$ & $\mathrm{r}, \mathrm{H}$ & odel\#105 & 1, NIs & bi & & & \\
\hline Ref, $d L$ & $1 \mathrm{a}$ & $1 \mathrm{~b}$ & $2 \mathrm{a}$ & $2 \mathrm{~b}$ & $K, 1 \mathrm{a}$ & $K, 1 \mathrm{~b}$ & $K, 2 \mathrm{a}$ & $K, 2 \mathrm{~b}$ & average \\
\hline $\begin{array}{c}\mathrm{mm} \\
551.813\end{array}$ & $\begin{array}{c}\text { Pulse } \\
27586\end{array}$ & $\begin{array}{c}\text { Pulse } \\
27586\end{array}$ & $\begin{array}{l}\text { Pulse } \\
27598\end{array}$ & $\begin{array}{c}\text { Pulse } \\
27596\end{array}$ & $\begin{array}{c}\mathrm{p} / \mathrm{mm} \\
49.9916\end{array}$ & $\begin{array}{c}\mathrm{p} / \mathrm{mm} \\
49.9916\end{array}$ & $\begin{array}{c}\mathrm{p} / \mathrm{mm} \\
50.0134\end{array}$ & $\begin{array}{c}\mathrm{p} / \mathrm{mm} \\
50.0097\end{array}$ & \\
\hline 550.370 & 27514 & 27514 & 27524 & 27524 & 49.9918 & 49.9918 & 50.0100 & 50.0100 & \\
\hline 549.345 & 27462 & 27462 & 27475 & 27473 & 49.9905 & 49.9905 & 50.0141 & 50.0105 & \\
\hline 550.951 & 27543 & 27543 & 27553 & 27553 & 49.9918 & 49.9918 & 50.0099 & 50.0099 & \\
\hline & & & & mean $K_{E R}$ & 49.9914 & 49.9914 & 50.0118 & 50.0100 & 50.001 \\
\hline & & & & std unc & 0.0003 & 0.0003 & 0.0011 & 0.0002 & 0.0005 \\
\hline & & & & & & & Ref unc & & $6.3 \mathrm{E}-06$ \\
\hline & & & & & & & Combine & unc & 0.0005 \\
\hline
\end{tabular}

Table B6. Diameter, piston displacement, and volume measurement uncertainties.

\begin{tabular}{|ccccccccc|}
\hline \multicolumn{7}{c|}{$D=D_{R}\left(1+\alpha_{s} \Delta T_{D R}\right)$} \\
$D_{R}[\mathrm{~cm}]$ & mean & $u_{A}$ & $u_{B}$ & $\% u_{A}$ & $\% u_{B}$ & $c_{i D}$ & $D \% u_{A}$ & $D \% u_{B}$ \\
$\alpha_{S}[1 / \mathrm{K}]$ & $1.7 \mathrm{E}-05$ & $0.0 \mathrm{E}+00$ & $3.4 \mathrm{E}-07$ & $0.0 \mathrm{E}+00$ & 2.0000 & $-1.4 \mathrm{E}-06$ & $0.0 \mathrm{E}+00$ & $-2.9 \mathrm{E}-06$ \\
& & & & & - & $-1.4 \mathrm{E}-$ & & \\
$\Delta T_{D R}[\mathrm{~K}]$ & -0.084 & 0.010 & 0.024 & -11.955 & 28.6828 & 06 & $1.7 \mathrm{E}-05$ & $4.1 \mathrm{E}-05$ \\
$D[\mathrm{~cm}]$ & 7.6188 & $1.3 \mathrm{E}-06$ & 0.0001 & & & & $1.7 \mathrm{E}-05$ & 0.0008
\end{tabular}

\begin{tabular}{cccccccccc}
\hline \multicolumn{8}{c|}{$d=d_{R}\left(1+\alpha_{s} \Delta T_{d R}\right)$} \\
\hline & mean & $u_{A}$ & $u_{B}$ & $\% u_{A}$ & $\% u_{B}$ & $c_{i d}$ & $d \% u_{A}$ & $d \% u_{B}$ \\
$d_{R}[\mathrm{~cm}]$ & 2.5441 & $0.0 \mathrm{E}+00$ & 0.0001 & $0.0 \mathrm{E}+00$ & 0.0022 & 1.00000 & $0.0 \mathrm{E}+00$ & 0.0022 \\
& & & & & & $-3.5 \mathrm{E}-$ & & \\
$\alpha_{s}[1 / \mathrm{K}]$ & $1.7 \mathrm{E}-05$ & $0.0 \mathrm{E}+00$ & $3.4 \mathrm{E}-07$ & $0.0 \mathrm{E}+00$ & 2.0000 & 06 & $0.0 \mathrm{E}+00$ & $-7.0 \mathrm{E}-06$ \\
& & & & & - & $-3.5 \mathrm{E}-$ & & \\
$\Delta T_{d R}[\mathrm{~K}]$ & -0.206 & 0.010 & 0.024 & -4.875 & 11.6959 & 06 & $1.7 \mathrm{E}-05$ & $4.1 \mathrm{E}-05$ \\
$d[\mathrm{~cm}]$ & 2.5441 & $4.3 \mathrm{E}-07$ & 0.0001 & & & & $1.7 \mathrm{E}-05$ & 0.0022
\end{tabular}




\begin{tabular}{cccccccccc|}
\hline \multicolumn{8}{c|}{$K_{E}=K_{R} /\left(1+\alpha_{E} \Delta T_{E R}\right)$} \\
$K_{R}[\mathrm{p} / \mathrm{cm}]$ & mean & $u_{A}$ & $u_{B}$ & $\% u_{A}$ & $\% u_{B}$ & $c_{i K}$ & $K \% u_{A}$ & $K \% u_{B}$ \\
& 500.01 & $0.0 \mathrm{E}+00$ & 0.0048 & $0.0 \mathrm{E}+00$ & 0.0010 & 1.0000 & $0.0 \mathrm{E}+00$ & 0.0010 \\
$\alpha_{E}[1 / \mathrm{K}]$ & $8.0 \mathrm{E}-06$ & $0.0 \mathrm{E}+00$ & $1.0 \mathrm{E}-06$ & $0.0 \mathrm{E}+00$ & 12.5000 & 06 & $0.0 \mathrm{E}+00$ & $-1.0 \mathrm{E}-04$ \\
& & & & & & $-8.0 \mathrm{E}-$ & & \\
$\Delta T_{E R}[\mathrm{~K}]$ & 1.000 & 0.010 & 0.033 & 1.008 & 3.257 & 06 & $-8.1 \mathrm{E}-06$ & $-2.6 \mathrm{E}-05$ \\
$K_{E}[\mathrm{p} / \mathrm{cm}]$ & 500.02 & $4.0 \mathrm{E}-05$ & 0.0048 & & & & $8.1 \mathrm{E}-06$ & 0.0010
\end{tabular}

\begin{tabular}{ccccccccc|}
\hline \multicolumn{7}{c|}{$\Delta V p=\pi\left(D^{2}-d^{2}\right) / 4 L=\pi\left(D^{2}-d^{2}\right) / 4 N_{E} / K_{E}$} \\
\hline mean & $u_{A}$ & $u_{B}$ & $\% u_{A}$ & $\% u_{B}$ & $c_{i V}$ & $V \% u_{A}$ & $V \% u_{B}$ \\
$D[\mathrm{~cm}]$ & 7.6188 & $1.3 \mathrm{E}-06$ & 0.0001 & 0.0000 & 0.0008 & 2.25101 & $3.8 \mathrm{E}-05$ & 0.0018 \\
$d[\mathrm{~cm}]$ & 2.5441 & $4.3 \mathrm{E}-07$ & 0.0001 & 0.0000 & 0.0022 & 0.25101 & $-4.3 \mathrm{E}-06$ & -0.0006 \\
$K_{E}[\mathrm{p} / \mathrm{cm}]$ & 500.02 & $4.0 \mathrm{E}-05$ & 0.0048 & $8.1 \mathrm{E}-06$ & 0.0010 & -1.000 & $-8.1 \mathrm{E}-06$ & -0.0010 \\
$\Delta V_{P}\left[\mathrm{~cm}^{3}\right]$ & 1514.88 & 0.0006 & 0.0317 & & & & $3.9 \mathrm{E}-05$ & 0.0021
\end{tabular}

\begin{tabular}{|c|c|c|c|c|c|c|c|c|}
\hline \multicolumn{9}{|c|}{$V_{C V}=\pi d_{C V}^{2} / 4 l_{C V}$} \\
\hline & mean & $u_{A}$ & $u_{B}$ & $\% u_{A}$ & $\% u_{B}$ & $c_{i V}$ & $V \% u_{A}$ & $V \% u_{B}$ \\
\hline$d_{C V}[\mathrm{~cm}]$ & 0.9525 & $0.0 \mathrm{E}+00$ & 0.0508 & $0.0 \mathrm{E}+00$ & 5.3333 & 2 & $0.0 \mathrm{E}+00$ & 10.6667 \\
\hline$l_{C V}[\mathrm{~cm}]$ & 274.32 & $0.0 \mathrm{E}+00$ & 12.700 & $0.0 \mathrm{E}+00$ & 4.6296 & 1 & $0.0 \mathrm{E}+00$ & 4.6296 \\
\hline$V c v\left[\mathrm{~cm}^{3}\right]$ & 195.47 & $0.0 \mathrm{E}+00$ & 22.729 & & & & $0.0 \mathrm{E}+00$ & 11.6280 \\
\hline
\end{tabular}


B3. Timing and counter uncertainties.

Table B7. Sample calibration data for two oscillators that are used for time and frequency measurements.

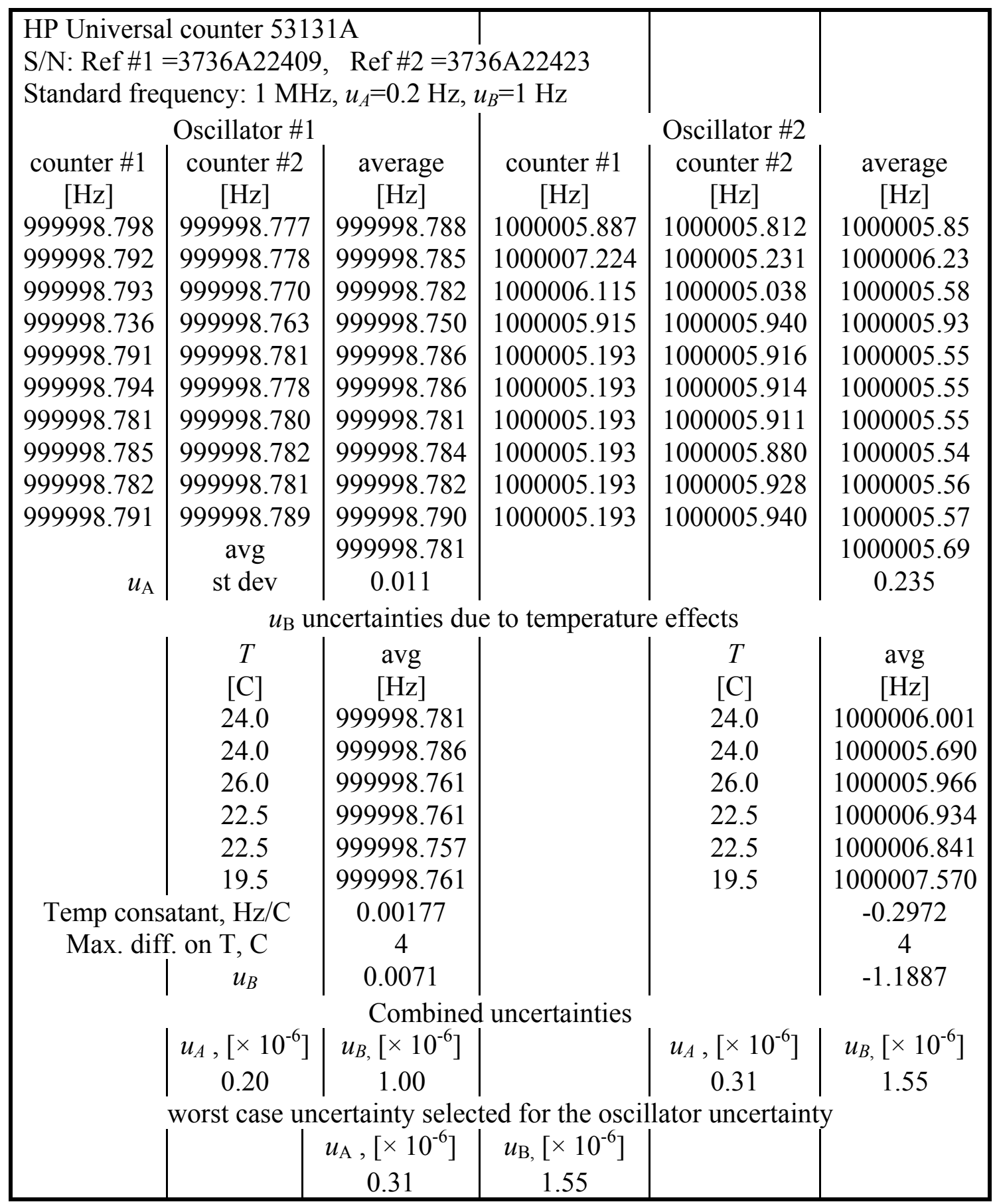


Table B8. Encoder timing uncertainty.

\begin{tabular}{ccccccccc|}
\hline \multicolumn{8}{c|}{$t=\left(t_{1 A}+t_{1 B}+t_{2 A}+t_{2 B}\right) / 4$} \\
$t_{1 A}[\mathrm{~s}]$ & 30.000 & $1.0 \mathrm{E}-05$ & $4.7 \mathrm{E}-05$ & $3.4 \mathrm{E}-05$ & $1.6 \mathrm{E}-04$ & 0.25 & $8.6 \mathrm{E}-06$ & $3.9 \mathrm{E}-05$ \\
$t_{1 B}[\mathrm{~s}]$ & 30.000 & $1.0 \mathrm{E}-05$ & $4.7 \mathrm{E}-05$ & $3.4 \mathrm{E}-05$ & $1.6 \mathrm{E}-04$ & 0.25 & $8.6 \mathrm{E}-06$ & $3.9 \mathrm{E}-05$ \\
$t_{2 A}[\mathrm{~s}]$ & 30.000 & $1.0 \mathrm{E}-05$ & $4.7 \mathrm{E}-05$ & $3.4 \mathrm{E}-05$ & $1.6 \mathrm{E}-04$ & 0.25 & $8.6 \mathrm{E}-06$ & $3.9 \mathrm{E}-05$ \\
$t_{2 B}[\mathrm{~s}]$ & 30.000 & $1.0 \mathrm{E}-05$ & $4.7 \mathrm{E}-05$ & $3.4 \mathrm{E}-05$ & $1.6 \mathrm{E}-04$ & 0.25 & $8.6 \mathrm{E}-06$ & $3.9 \mathrm{E}-05$ \\
$t[\mathrm{~s}]$ & 30.000 & $5.2 \mathrm{E}-06$ & $3.3 \mathrm{E}-05$ & & & & $1.7 \mathrm{E}-05$ & $1.1 \mathrm{E}-04$
\end{tabular}

Table B9. Properties of hydrocarbon liquid fluid, MIL-C-7024C.

\begin{tabular}{|c|c|c|c|c|}
\hline \multicolumn{3}{|c|}{ Fluid density } & \multicolumn{2}{|c|}{ Fluid kinematic viscosity } \\
\hline$T$ & $\rho$ & & $T$ & $v$ \\
\hline [C] & {$\left[\mathrm{kg} / \mathrm{m}^{3}\right]$} & & [C] & [cs] \\
\hline 15.14 & 788.0 & & 20.03 & 1.248 \\
\hline 19.98 & 784.4 & & 20.03 & 1.254 \\
\hline 24.95 & 780.5 & & 24.99 & 1.164 \\
\hline \multirow[t]{8}{*}{29.70} & 776.8 & & 24.99 & 1.164 \\
\hline & & & 29.95 & 1.088 \\
\hline & & & 29.95 & 1.089 \\
\hline & & & 29.95 & 1.088 \\
\hline & & & 29.95 & 1.066 \\
\hline & & & 29.95 & 1.061 \\
\hline & & & 15.08 & 1.344 \\
\hline & & & 15.08 & 1.344 \\
\hline \multicolumn{3}{|c|}{$\rho=\rho_{o}(1+\alpha \Delta T)$} & \multicolumn{2}{|c|}{$v=v_{o}+c T$} \\
\hline$\rho_{o}$ & $\alpha$ & $T_{r}$ & $v_{o}$ & $c$ \\
\hline$\left[\mathrm{kg} / \mathrm{m}^{3]}\right.$ & {$[1 / \mathrm{C}]$} & [C] & [cs] & {$[\mathrm{cs} / \mathrm{C}]$} \\
\hline 784.3 & 0.0010 & 20 & 1.610 & -0.018 \\
\hline
\end{tabular}




\title{
Appendix C: Sample Calibration Report
}

\section{REPORT OF CALIBRATION}

\author{
For A Dual Turbine Flowmeter
}

April 28, 2005

Manufacturer: Turbine Meters Inc.

Serial Number: ABC123

submitted by

Customer Co. Name

Gaithersburg, MD

(Purchase Order Number 123456, dated April 1, 2005)

The flowmeter identified above was calibrated using the NIST 2 L Hydrocarbon Liquid Flow System (HLFS). This facility calculates volumetric flow by measuring the time required for a piston to displace a certain volume within a cylinder. Averages of the output of the meter under test were gathered over the same intervals that the volumetric flow was calculated from the primary standard.

A dimensionless presentation of the calibration data facilitates accurate flow measurements by the flowmeter user even when the conditions of usage (liquid type, temperature) differ from the conditions during calibration. For a turbine flowmeter, the suggested parameters are the 
dimensionless meter factor ${ }^{1}$, i.e., the Strouhal number, $S t$, and for the meter frequency, the Roshko number, Ro. These are written,

$$
\begin{aligned}
& S t=\frac{f d}{U_{M}}=\frac{\pi K d^{3}}{4}=\frac{\pi f d^{3}}{4 Q_{M}}, \text { and } \\
& R o=\frac{f d^{2}}{v},
\end{aligned}
$$

where, in compatible units:

$$
\begin{aligned}
& K=f / Q_{M}, \text { the meter factor in pulses per unit volume, } \\
& d=\text { the diameter of the meter (nominal diameter as specified by the manufactuere) } \\
& Q_{M}=\text { volumetric flow, } \\
& U_{M}=4 Q_{M} / \pi d^{2} \text {, average flow velocity in the meter, } \\
& f=\text { the frequency of the meter output signal, and } \\
& v=\text { the kinematic viscosity of the fluid. }
\end{aligned}
$$

The test liquid is MIL-C-7024C fluid (also know as Stoddard or Type 2 solvent) - a surrogate liquid for JP-4 and JP-5 jet fuels. The densities and viscosities of the liquid used in the system were calibrated by NIST to have the following dependencies on temperature, assuming pressure effects to be negligible:

$$
\begin{aligned}
& \rho=782.614 *(1-0.000984 *(T-22.2)), \text { and } \\
& \nu=1.6097-0.01777 * T,
\end{aligned}
$$

where,

$\rho=$ the fluid density, in $\mathrm{kg} / \mathrm{m} 3$,

$v=$ the kinematic viscosity of the fluid, in centistokes, and

\footnotetext{
${ }^{1}$ G. E. Mattingly, "The Characterization of a Piston Displacement-Type Flowmeter Calibration Facility and the Calibration and Use of Pulsed Output Type Flowmeters,” NIST J. of Res., 975 (1992)
} 
$T=$ the temperature of the fluid, in ${ }^{\circ} \mathrm{C}$.

The diameter of the flowmeter used in calculations of the $R o$ and $S t$ is $12.5 \mathrm{~mm}$, the nominal diameter specified by the manufacturer. The dual turbine flowmeter was installed in a test section with 100 diameters of straight pipe upstream and 75 diameters of straight pipe downstream. A photograph of the installation is shown in Figure 1.

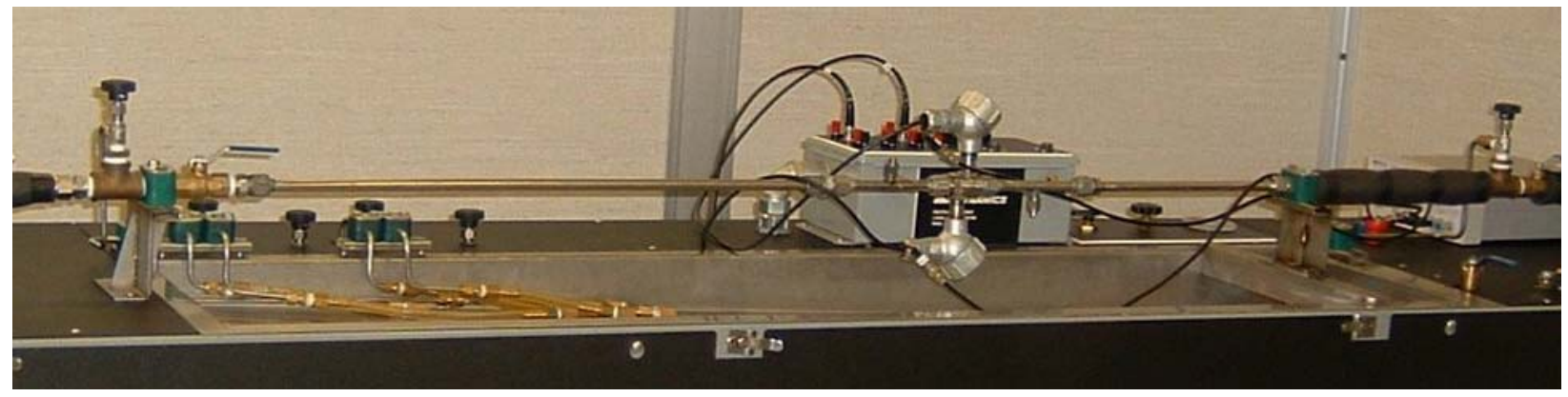

Figure 1 A photograph of the flowmeter installation.

An analysis was performed to assess the uncertainty of the calibration results obtained for the meter under test. ${ }^{2,3,4}$ The process involves identifying the equations used in calculating the calibration result (measurand) so that the sensitivity of the result to uncertainties in the input quantities can be evaluated. The approximately $67 \%$ confidence level uncertainty of each of the input quantities is determined, weighted by its sensitivity, and combined with the other uncertainty components by root-sum-square to arrive at a combined uncertainty $\left(U_{c}\right)$. The combined uncertainty is multiplied by a coverage factor of 2.0 to arrive at an expanded uncertainty $\left(U_{e}\right)$ of the measurand with approximately $95 \%$ confidence level.

As described in the references, if one considers a generic basis equation for the measurement process, which has an output, $y$, based on $N$ input quantities, $x_{i}$,

2 International Organization for Standardization, Guide to the Expression of Uncertainty in Measurement, Switzerland, 1996 edition.

3 Taylor, B. N. and Kuyatt, C. E., Guidelines for Evaluating and Expressing the Uncertainty of NIST Measurement Results, NIST TN 1297, 1994 edition.

${ }^{4}$ Coleman, H. W. and Steele, W. G., Experimentation and Uncertainty Analysis for Engineers, John Wiley and Sons, 2nd ed., 1999. 


$$
y=y\left(x_{1}, x_{2}, \ldots, x_{N}\right)
$$

and all uncertainty components are uncorrelated, the normalized expanded uncertainty is given by,

$$
\frac{U_{e}(y)}{y}=k \frac{U_{c}(y)}{y}=k \sqrt{\sum_{i=1}^{N} s_{i}^{2}\left(\frac{u\left(x_{i}\right)}{x_{i}}\right)^{2}}
$$

In the normalized expanded uncertainty equation, the $u\left(x_{i}\right)^{\prime} s$ are the standard uncertainties of each input, and $s_{i}{ }^{\prime} s$ are their associated sensitivity coefficients, given by,

$$
s_{i}=\frac{\partial y}{\partial x_{i}} \frac{x_{i}}{y}
$$

The normalized expanded uncertainty equation is convenient since it permits the usage of relative uncertainties (in fractional or percentage forms) and of dimensionless sensitivity coefficients. The dimensionless sensitivity coefficients can often be obtained by inspection since for a linear function they have a magnitude of unity.

For this calibration, the uncertainty of the Strouhal number has components due to the measurement of the actual volumetric flow at the meter under test $u\left(Q_{M}\right)$, the frequency output of the meter under test $U(f)$, and the reproducibility of the test $u(R)$, all of which have sensitivity coefficients of 1 . The expanded uncertainty of the volumetric flow from the HLFS has uncertainty of $0.001 \%{ }^{5}$ which leads to a standard uncertainty of $0.005 \%$.

The relative standard uncertainty of the frequency measurements is less than $2 \times 10^{-6}$ based on analysis of calibration records and can be considered negligible. The present uncertainty analysis does not include an uncertainty from the nominal diameter of the MUT.

${ }^{5}$ T. T. Yeh, P.I. Espina, G.E. Mattingly, and N.R. Briggs, An UncertaintyAnalysis of a NIST Hydrocarbon Liquid Flow Calibration Facility, Proceedings of HT/FED'04, HT-FED2004-56790, 2004 Heat Transfer/Fluids Engineering Summer Conference, Charlotte, North Carolina, USA, July 11-15, 2004. 
A normal flow calibration performed by the NIST Fluid Metrology Group consists of six flows spread over the range of the flowmeter. A flowmeter is normally calibrated at $10 \%, 20 \%, 35 \%$, $50 \%, 75 \%$, and $100 \%$ of the meter full scale. At each of these flow set points, five flow measurements are made. The same set point flows are tested on a second occasion, but the flows are tested in decreasing order instead of the increasing order of the first set. Therefore, the final data set consists of ten primary flow measurements made at six flow set points, i.e., 60 individual flow measurements. To measure the reproducibility ${ }^{6}$ of the test, the standard deviation of the Strouhal number at each of the nominal flows was used to calculate the relative standard uncertainty (the standard deviation divided by the mean and expressed as a percentage).

Using the values given above results in the expanded uncertainties listed in the following data tables and shown as error bars in the figures. Data for the upstream rotor (turbine 1) and the downstream rotor (turbine 2), as well as for their sum (turbine 1\&2) are shown.

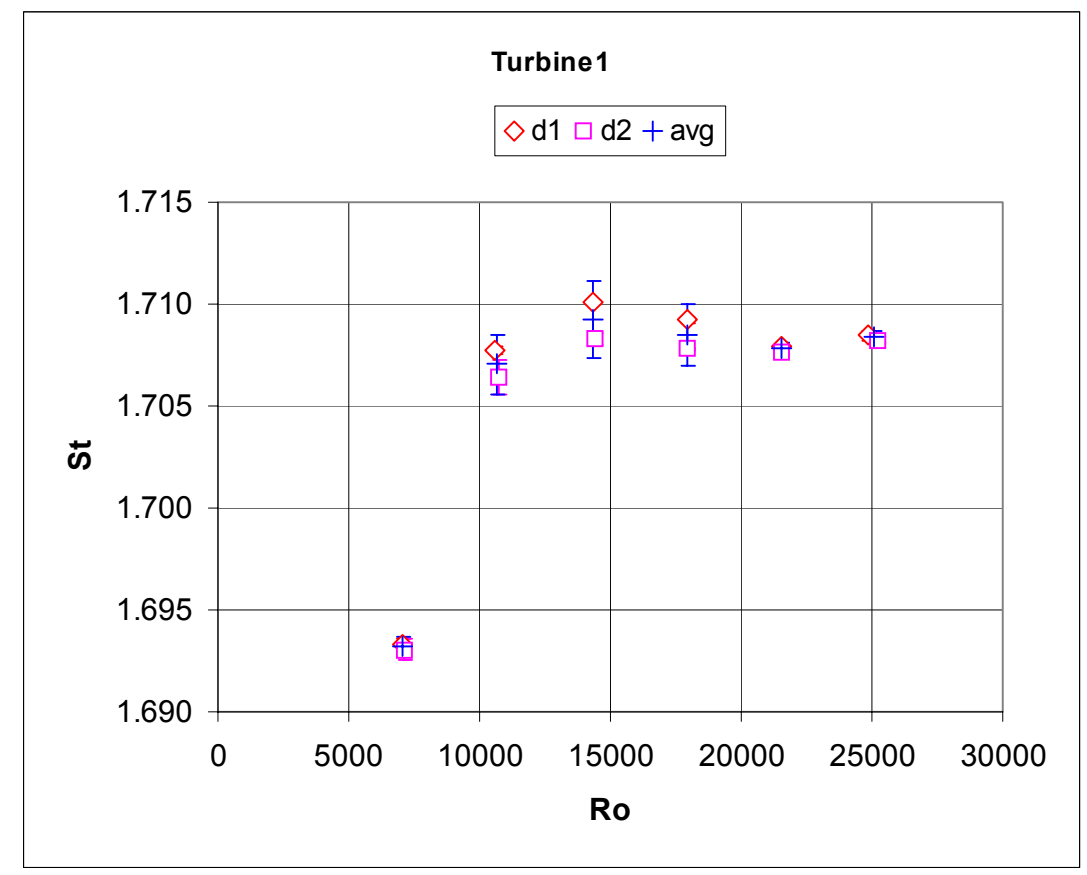

Figure 2. Strouhal number versus Roshko number for the upstream rotor (turbine 1). Symbols "d1" and "d2" represent the averages of data collected on two different occasions, while "avg" is the results for Day-1\&2, reproducibility.

\footnotetext{
${ }^{6}$ Reproducibility is herein defined as the closeness of agreement between measurements with the flow changed and then returned to the same nominal value.
} 


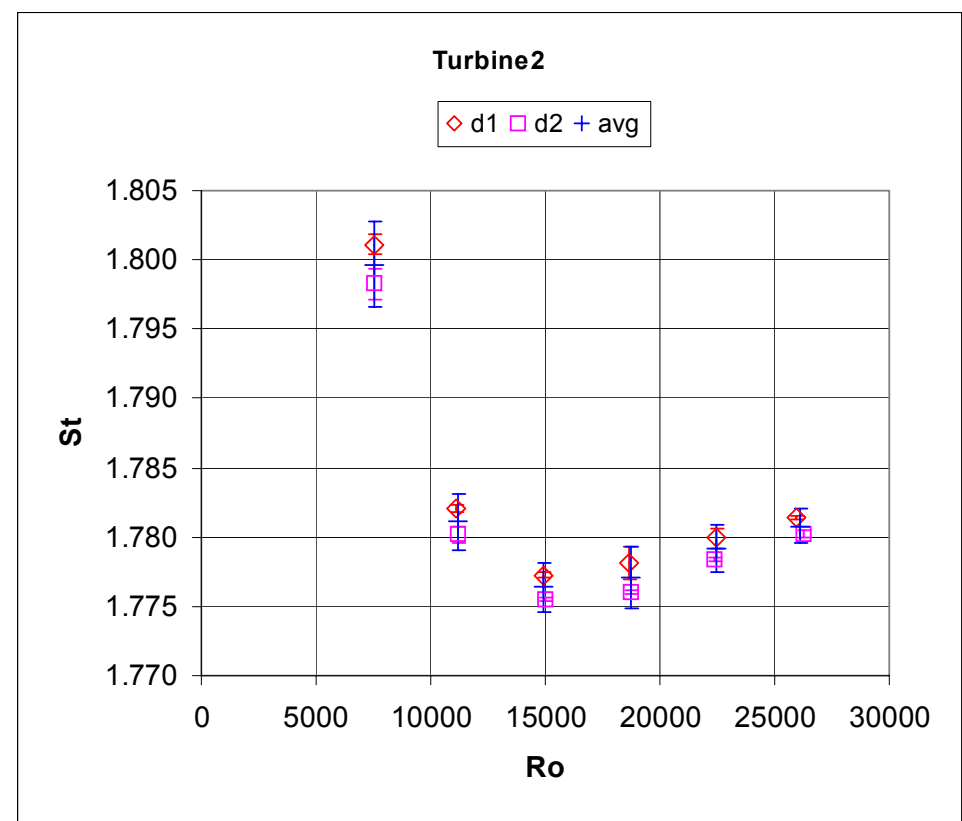

Figure 3. Strouhal number versus Roshko number for the downstream rotor (turbine 2). Symbols "d1" and "d2" represent the averages of data collected on two different occasions, while "avg" is the results for Day-1\&2, reproducibility.

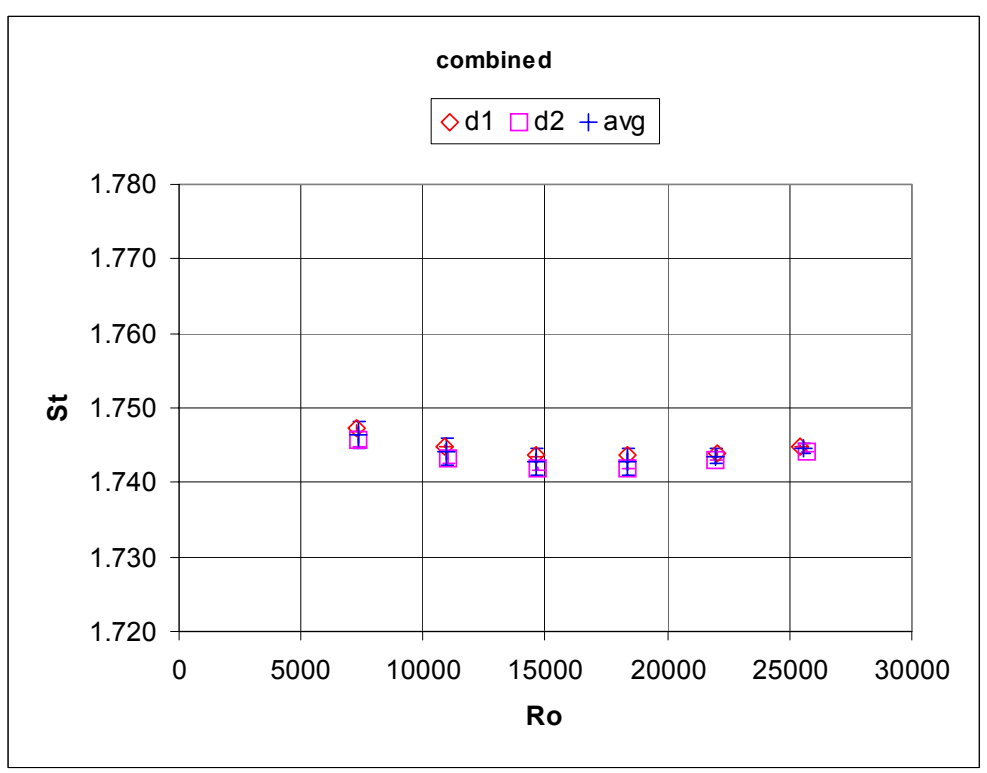

Figure 4. Strouhal number versus Roshko number for the sum of the upstream and downstream rotor (turbine 1\&2). Symbols "d1" and "d2" represent the averages of data collected on two different occasions, while "avg" is the results for Day1\&2, reproducibility.

Table 1. Statistical Results and Uncertainties for dual turbine flowmeter. 


\begin{tabular}{|c|c|c|c|c|c|c|c|c|}
\hline $\begin{array}{c}T_{M} \\
{\left[{ }^{\circ} \mathrm{C}\right]}\end{array}$ & {$[\mathrm{kg} / \mathrm{L}]$} & {$\left[\mathrm{cm}^{2} / \mathrm{s}\right]$} & $\underset{[\mathrm{L} / \mathrm{min}]}{\boldsymbol{Q}_{M}}$ & $\begin{array}{c}\boldsymbol{f} \\
{[\mathrm{Hz}]}\end{array}$ & $\begin{array}{r}\mathbf{R}_{\mathbf{0}} \\
{\left[d^{2} f l\right.}\end{array}$ & {$\left[\begin{array}{c}\mathbf{S}_{\mathbf{t}} \\
{\left[\begin{array}{l}d^{2} f / 4 Q_{M}\end{array}\right]}\end{array}\right.$} & $\begin{array}{c}\text { Std Dev } \\
{[\%]}\end{array}$ & $\begin{array}{l}U_{k=2} \\
{[\%]}\end{array}$ \\
\hline \multicolumn{9}{|c|}{ Turbine 1} \\
\hline 22.42 & 782.8 & 0.0121 & 1.518 & 212.96 & 7089.2 & 1.6932 & 0.0244 & 0.0487 \\
\hline 22.39 & 782.8 & 0.0121 & 2.271 & 321.25 & 10689.6 & 1.7071 & 0.0740 & 0.1480 \\
\hline 22.35 & 782.7 & 0.0121 & 3.051 & 432.16 & 14370.0 & 1.7092 & 0.0955 & 0.1910 \\
\hline 22.40 & 782.8 & 0.0121 & 3.814 & 539.99 & 17969.1 & 1.7085 & 0.0754 & 0.1508 \\
\hline 22.40 & 782.8 & 0.0121 & 4.573 & 647.23 & 21537.9 & 1.7078 & 0.0159 & 0.0317 \\
\hline 22.44 & 782.8 & 0.0121 & 5.311 & 751.93 & 25037.0 & 1.7084 & 0.0144 & 0.0288 \\
\hline \multicolumn{9}{|c|}{ Turbine 2} \\
\hline 22.42 & 782.8 & 0.0121 & 1.518 & 226.36 & 7535.1 & 1.7997 & 0.1567 & 0.3134 \\
\hline 22.39 & 782.8 & 0.0121 & 2.271 & 335.19 & 11153.4 & 1.7811 & 0.1024 & 0.2048 \\
\hline 22.35 & 782.7 & 0.0121 & 3.051 & 449.14 & 14934.6 & 1.7764 & 0.0903 & 0.1805 \\
\hline 22.40 & 782.8 & 0.0121 & 3.814 & 561.67 & 18690.5 & 1.7771 & 0.1135 & 0.2271 \\
\hline 22.40 & 782.8 & 0.0121 & 4.573 & 674.26 & 22437.4 & 1.7791 & 0.0856 & 0.1712 \\
\hline 22.44 & 782.8 & 0.0121 & 5.311 & 783.81 & 26098.5 & 1.7808 & 0.0639 & 0.1278 \\
\hline \multicolumn{9}{|c|}{ Turbine $1 \& 2$} \\
\hline 22.42 & 782.8 & 0.0121 & 1.518 & 219.66 & 7312.2 & 1.7464 & 0.0884 & 0.1767 \\
\hline 22.39 & 782.8 & 0.0121 & 2.271 & 328.22 & 10921.5 & 1.7441 & 0.0874 & 0.1749 \\
\hline 22.35 & 782.7 & 0.0121 & 3.051 & 440.65 & 14652.3 & 1.7428 & 0.0927 & 0.1855 \\
\hline 22.40 & 782.8 & 0.0121 & 3.814 & 550.83 & 18329.8 & 1.7428 & 0.0929 & 0.1857 \\
\hline 22.40 & 782.8 & 0.0121 & 4.573 & 660.74 & 21987.7 & 1.7435 & 0.0502 & 0.1005 \\
\hline 22.44 & 782.8 & 0.0121 & 5.311 & 767.87 & 25567.7 & 1.7446 & 0.0377 & 0.0755 \\
\hline
\end{tabular}

For the Director,

National Institute of Standards and Technology

Group Leader, Fluid Metrology Group

Process Measurements Division

Chemical Science and Technology Laboratory 